\title{
Escherization with Large Deformations Based on As-Rigid-As-Possible Shape Modeling
}

\author{
YUICHI NAGATA, Tokushima University, Japan \\ SHINJI IMAHORI, Chuo University, Japan
}

Escher tiling is well known as a tiling that consists of one or a few recognizable figures, such as animals. The Escherization problem involves finding the most similar shape to a given goal figure that can tile the plane. However, it is easy to imagine that there is no similar tile shape for complex goal shapes. This article devises a method for finding a satisfactory tile shape in such a situation. To obtain a satisfactory tile shape, the tile shape is generated by deforming the goal shape to a considerable extent while retaining the characteristics of the original shape. To achieve this, both goal and tile shapes are represented as triangular meshes to consider not only the contours but also the internal similarity of the shapes. To measure the naturalness of the deformation, energy functions based on traditional as-rigid-as-possible shape modeling are incorporated into a recently developed framework of the exhaustive search of the templates for the Escherization problem. The developed algorithms find satisfactory tile shapes with natural deformations for fairly complex goal shapes.

CCS Concepts: • Computing methodologies $\rightarrow$ Mesh geometry models; $\bullet$ Theory of computation $\rightarrow$ Computational geometry;

Additional Key Words and Phrases: Escherization, Escher tiling, as-rigidas-possible deformation, tessellations, procrustes distance

ACM Reference format:

Yuichi Nagata and Shinji Imahori. 2021. Escherization with Large Deformations Based on As-Rigid-As-Possible Shape Modeling. ACM Trans. Graph. 41, 2, Article 11 (November 2021), 16 pages.

https://doi.org/10.1145/3487017

\section{INTRODUCTION}

A tiling is a collection of shapes that cover the plane without any gaps or overlaps. Tilings appear everywhere in everyday life, from simple rectangular sequences to decorative and artistic ones. Artistic tilings created by the Dutch artist M. C. Escher are well known for their interesting appearance, where a tiling consists of one or a few recognizable figures, such as animals. Such artistic tilings are known as Escher tilings. Designing an artistic Escher-like tiling is a highly intellectual task because it is difficult to create meaningful tile shapes that satisfy the constraints to enable tiling. Therefore,

This work was supported by JSPS KAKENHI Grant Number 20K11695.

Authors' addresses: Y. Nagata, Tokushima University, 2-1 Minami-jousanjima, Tokushima-shi, Tokushima, 770-8506, Japan; email: nagata@is.tokushima-u.ac.jp; S. Imahori, Chuo University, 1-13-27 Kasuga, Bunkyo-ku, Tokyo, 112-8551, Japan; email imahori@ise.chuo-u.ac.jp.

Permission to make digital or hard copies of all or part of this work for personal or classroom use is granted without fee provided that copies are not made or distributed for profit or commercial advantage and that copies bear this notice and the full citation on the first page. Copyrights for components of this work owned by others than ACM must be honored. Abstracting with credit is permitted. To copy otherwise, or republish, to post on servers or to redistribute to lists, requires prior specific permission and/or a fee. Request permissions from permissions@acm.org.

(c) 2021 Association for Computing Machinery.

0730-0301/2021/11-ART11 \$15.00

https://doi.org/10.1145/3487017

several drawing support tools for Escher-like tiling have been proposed [Ono et al. 2014, 2015; Sugihara 2010].

To generate Escher-like tilings automatically, Kaplan and Salesin [2000] formulated the following optimization problem.

Escherization problem: Given a closed plane figure $S$ (goal shape), find a closed figure $T$ such that

(1) $T$ is as close as possible to $S$, and

(2) copies of $T$ fit together to form a tiling of the plane.

To formulate this problem, they introduced parameterizations of possible tile shapes for 93 types of isohedral tilings, which are a class of tiling that is sufficiently flexible to express tiling patterns. For each isohedral type, all possible tile shapes can be generated by deforming a template, which is represented by a polygon with at most six vertices, under the specified constraints. To measure the similarity between two shapes, $S$ and $T$, they employed a metric developed by Arkin et al. [1991], which is insensitive to rigid transformation and scaling of the shapes. Because the formulated problem cannot be solved analytically, they developed a simulated annealing algorithm for solving the formulated problem and successfully found satisfactory tile shapes for convex or nearly convex goal shapes.

Koizumi and Sugihara [2011] formulated the Escherization problem so that the formulated problem can be solved analytically. In this formulation, the goal and tile shapes were represented as $n$ point polygons. For each isohedral type, possible tile shapes were represented by $n$ points located on the template, which allows the possible tile shapes to be parameterized by a linear model. The Procrustes distance [Werman and Weinshall 1995] was employed to measure the similarity between the two polygons. The Procrustes distance is a rotation- and scale-invariant distance metric calculated based on the difference in the positions of the corresponding points between the two polygons. Under this formulation, the Escherization problem is reduced to a maximum eigenvalue problem. Koizumi and Sugihara's method performed well for both convex and non-convex goal shapes.

In Koizumi and Sugihara's original formulation, only one of the possible configurations of the template was considered for each isohedral type, where a huge number of templates can be configured depending on how the $n$ points are assigned to the edges of the template (see Figure 4). Later, Imahori and Sakai [2013] considered all possible configurations of the templates. This extension allows considerable flexibility in the possible tile shapes and, in principle, improves the quality of the obtained tile shapes. However, an exhaustive search of the templates (EST) is computationally impractical because the number of all possible template configurations is very large. As a compromise, they proposed a local 
search-based algorithm to search for only promising configurations of the templates. Recently, Nagata and Imahori [2020] developed an efficient algorithm to perform EST in a reasonable computation time.

EST fully exploits the potential of Koizumi and Sugihara's formulation of the Escherization problem. However, it is still difficult to obtain satisfactory tile shapes for fairly complex goal shapes. This is a natural consequence because we can easily imagine that there exists no shape that can tile the plane and is similar to the goal shape. In such a situation, tile shapes should be generated by deforming the goal shape to a considerable extent while retaining the characteristics of the original shape in order to obtain a satisfactory tile shape.

Our motivation here is similar to the ones in the layout or packing problem. In a typical layout problem, a given domain is filled with pre-specified geometric elements, or all the pre-specified geometric elements are arranged in a given domain. In both the cases, gaps between elements may or may not be allowed. In addition, elements may or may not be allowed to deform. The deformable setting is similar to the problem being addressed. Layout problems belonging to this category can be found in jigsaw image mosaics [Kim and Pellacini 2002], where mosaic elements are allowed to deform to achieve a more visually pleasing result. Peng et al. [2014] developed a widely applicable framework for tiling a domain with a set of deformable templates, each of which defines the permissible shapes for an element. Other examples that are similar to the Escherization problem are in Kwan et al. [2016], Saputra et al. [2018], and Zou et al. [2016], where a container shape is filled with a variety of deformable geometric elements such as animals and letters. In these examples, deformation plays a vital role in obtaining desirable results, for example, with equal spacing between elements. An essential difference of the Escherization problem from the layout problems described above is that all geometric elements (tile pieces) must be deformed in exactly the same way, making it challenging to obtain desirable results.

To achieve our goal in the Escherization problem, a distance function that can measure the naturalness of the deformation between the tile and goal shapes must be applied. For this purpose, we can borrow the concepts from the research field of 3D surface modeling and deformation. For example, in a typical surface editing task, a given 3D object represented as a mesh must be deformed in an intuitively plausible way under the constraints specified by the user. This task is inherently non-linear, but a variety of linear approaches [Alexa 2003; Lipman et al. 2004; Sorkine et al. 2004] have been proposed to achieve real-time interaction while avoiding artifacts resulting from linearization. Real-time non-linear approaches [Au et al. 2006; Igarashi et al. 2005; Sorkine and Alexa 2007] have also been proposed to account for the non-linearity of the problem. For a comprehensive survey, we refer the reader to Sorkine and Botsch [2009]. A common principle behind these approaches is to achieve the expected global deformations with intuitive local detail preservation. Among these techniques, we employ the as-rigid-as-possible (ARAP) deformation scheme [Sorkine and Alexa 2007] to construct a distance function suitable for the Escherization problem.

The contributions of this article are summarized as follows: (1) We demonstrate that our method can generate suitable tile

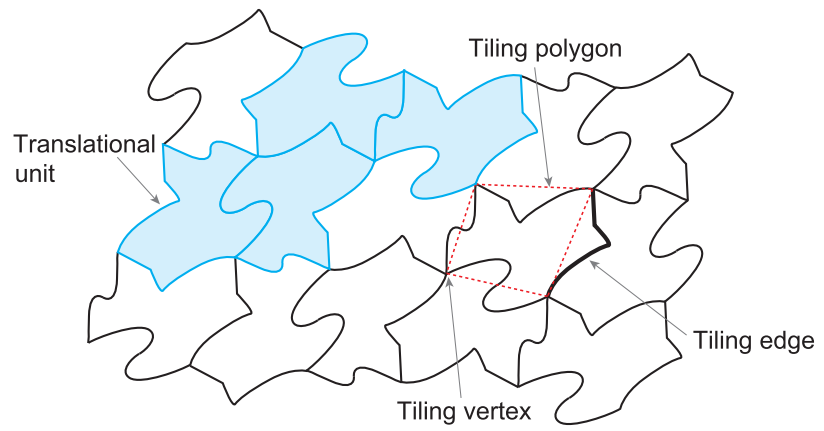

Fig. 1. Example of an isohedral tiling (IH53).

shapes for fairly complex goal shapes by deforming the goal shapes in intuitively plausible ways. (2) Although the ARAP deformation is a well-studied technique in 3D surface modeling and deformation, we demonstrate the effectiveness of this technique in an application area that is different from the traditional ones. (3) Our formulation makes it possible to use the ARAP deformation technique under a more complex situation in which some of the mesh's vertices are constrained by linear equations.

The remainder of this article is organized as follows. In Section 2, Koizumi and Sugihara's formulation for the Escherization problem and related works is described. In Section 3, the basis of the ARAP deformation is described. In Section 4, distance functions based on the ARAP deformation scheme are developed and incorporated into EST. In Section 5, an alternative approach to EST is proposed to reduce the computation time. In Section 6, experimental results are presented. In Section 7, limitations of the proposed method are described. In Section 8, contributions of this article are discussed.

\section{BACKGROUND}

\subsection{Isohedral Tilings}

Isohedral tiling is a class of tiling that consists of only one shape of tile and has translation symmetry, which means that a group of tiles consisting of one or several adjacent tiles repeats itself periodically over the whole tiling. Isohedral tilings can be classified into 93 different types [Grünbaum and Shephard 1987], which are named IH1, IH2, ... IH93, based on the adjacency relationship between tiles. Figure 1 shows an example of an isohedral tiling for IH53, where some technical terms [Kaplan and Salesin 2000] are defined. A point where the boundaries of three or more tiles meet is called a tiling vertex, whereas a curve where the boundaries of two tiles meet is called a tiling edge. A polygon constructed by connecting the tiling vertices of a tile is called a tiling polygon. The smallest unit consisting of one or several adjacent tiles that can tile the plane using only translations is called a translational unit.

The concept of tile templates [Kaplan 2009; Kaplan and Salesin 2000] is an intuitive way of characterizing the 93 isohedral types. Figure 2(a) shows the template of IH53; it represents a tiling polygon, from which all possible tile shapes can be generated by deforming the tiling edges. Tiling edges are classified into four types, which are specified by colored arrowheads depending on how they can be deformed as described below. 
(a)

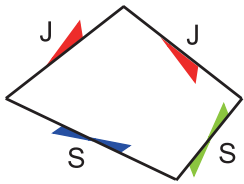

(b)

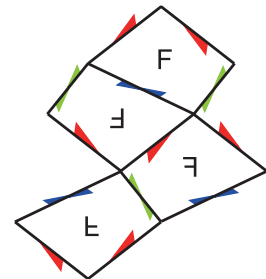

Fig. 2. (a) Template of IH53 and (b) a translational unit composed of the template.
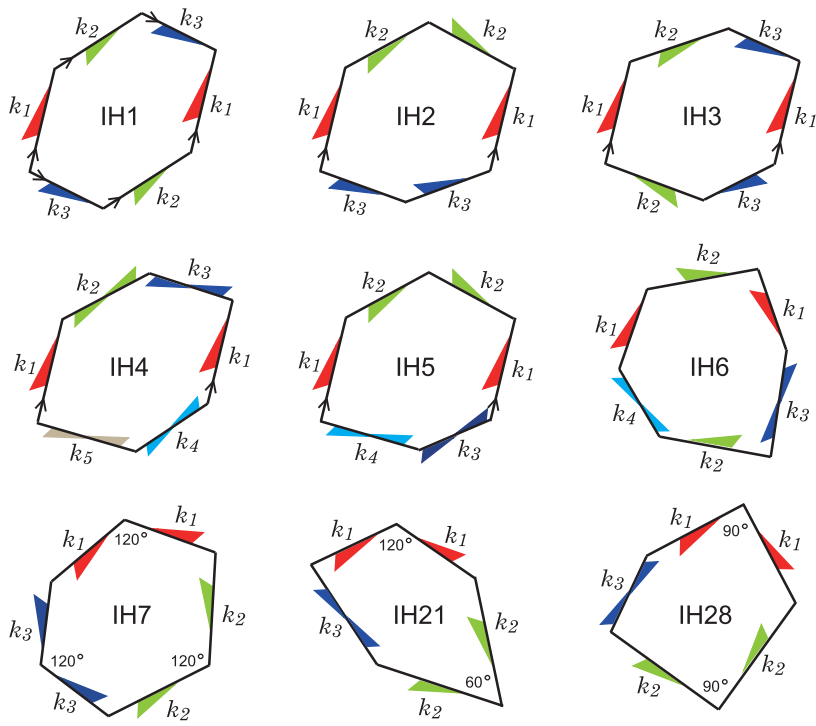

Fig. 3. Templates of the nine isohedral types used in this article. Two opposite $J$ edges marked with $\wedge$ are parallel to each other.

J edge: Can be freely deformed, but the corresponding $\mathrm{J}$ edge must have the same shape, as suggested by the arrowheads.

S edge: Can be freely deformed under the condition that it has rotational symmetry.

U edge: Can be freely deformed under the condition that it has reflectional symmetry.

I edge: Must be a straight line.

For each isohedral type, the adjacency relationship between tiles can be represented by arranging the template to form a translational unit (Figure 2(b)). For a more rigorous definition of the isohedral types, see Kaplan [2009]; Kaplan and Salesin [2000].

If the adjacency relationship between tiles is not considered, all possible tile shapes can be generated from the templates of the nine isohedral types [Schattschneider 2004] shown in Figure 3. Each of the templates of the 93 isohedral types can be obtained from one of the nine templates by removing one or more tiling edges and/or by replacing the $\mathrm{J}$ and $\mathrm{S}$ edges with $\mathrm{U}$ and I edges. For example, the template of IH53 is obtained from the template of IH5 by removing the two opposite J edges. We consider only the nine isohedral types in this article because all possible tile shapes can be generated from them.
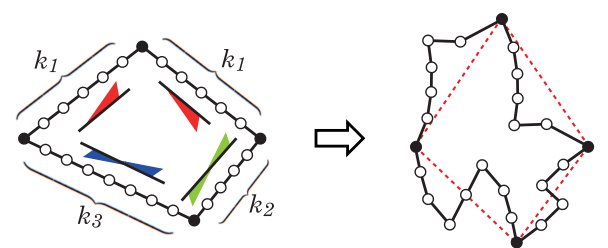

Fig. 4. Template of IH53 for a certain configuration of $\left(k_{1}, k_{2}, k_{3}\right)$ (left) and a tile shape obtained by deforming this template (right).

\subsection{Koizumi and Sugihara's Formulation and Extensions}

In Koizumi and Sugihara [2011], possible tile shapes are modeled as an $n$-point polygon $U$, which must form an isohedral tiling. For each isohedral type, possible positions of the $n$ points are parameterized by $n$ points placed on the template. For example, the template of IH53 is shown in Figure 4; exactly one point must be placed on every tiling vertex, and zero or more points are placed on each of the tiling edges, where the numbers of points placed on the tiling edges are denoted as $k_{1}, k_{2}$, and $k_{3}$. The parameters $k_{1}, k_{2}, \ldots$ are also displayed in the templates shown in Figure 3, where these numbers must be the same for J edges corresponding to each other. In Koizumi and Sugihara [2011], the same number of points was assigned to every tiling edge, i.e., $k_{1}=k_{2}=\cdots$. Subsequently, Imahori and Sakai [2013] extended this formulation by assigning different numbers of points to the tiling edges. The $n$ points on the template can move freely according to the constraints of the template (Figure 4).

The $n$ points on a template are numbered clockwise from 1 to $n$, starting from a tiling vertex. Let $\boldsymbol{u}_{i}=\left(x_{i}, y_{i}\right)^{\top}$ be the coordinates of the $i$ th point of the template, and the tile shape $U$ is represented as $\boldsymbol{u}=\left(x_{1}, y_{1}, \ldots, x_{n}, y_{n}\right)^{\top}$. For each of the templates, the constraint conditions imposed on the possible tile shapes are linear, and the possible values of $\boldsymbol{u}$ can be parameterized by

$$
\boldsymbol{u}=B \xi
$$

where $B\left(\in \mathbb{R}^{2 n \times m}\right)$ is a matrix, and $\xi\left(\in \mathbb{R}^{m}\right)$ is a parameter vector [Koizumi and Sugihara 2011]. Here, $B$ can be efficiently constructed in $O(n)$ time such that the column vectors are mutually orthonormal (i.e., $B^{\top} B$ is the identity matrix) [Nagata and Imahori 2020], although this requirement is not necessary after the next subsection. The goal shape is also represented as an $n$-point polygon $W$ (Figure 5(a)). The $n$ points are numbered clockwise from 1 to $n$. Let $\boldsymbol{w}_{i}=\left(x_{i}^{w}, y_{i}^{w}\right)^{\top}$ be the coordinates of the $i$ th point of $W$, and the goal shape $W$ is represented as $w=\left(x_{1}^{w}, y_{1}^{w}, \ldots, x_{n}^{w}, y_{n}^{w}\right)^{\top}$.

Koizumi and Sugihara [2011] employed the Procrustes distance [Werman and Weinshall 1995] to measure the similarity between the two polygons $U$ and $W$. In Nagata and Imahori [2020], a simplified version of the Procrustes distance was introduced in place of the original one. Let a matrix $V\left(\in \mathbb{R}^{2 n \times 2 n}\right)$ be defined by $V=$ $\boldsymbol{w} \boldsymbol{w}^{\top}+\boldsymbol{w}_{\boldsymbol{c}} \boldsymbol{w}_{\boldsymbol{c}}^{\top}$, where $\boldsymbol{w}_{\boldsymbol{c}}=\left(y_{1}^{w},-x_{1}^{w}, \ldots, y_{n}^{w},-x_{n}^{w}\right)^{\top}$. The simplified Procrustes distance $d_{P}(U, W)$ is defined and can be calculated as follows:

$$
\begin{aligned}
d_{P}^{2}(U, W) & =\min _{\theta} \sum_{i=1}^{n}\left\|R(\theta) \boldsymbol{u}_{i}-\boldsymbol{w}_{i}\right\|^{2} \\
& =\boldsymbol{u}^{\top} \boldsymbol{u}-2 \sqrt{\boldsymbol{u}^{\top} V \boldsymbol{u}}+\boldsymbol{w}^{\top} \boldsymbol{w},
\end{aligned}
$$


where $R(\theta)$ is the rotation matrix by angle $\theta$; the optimal value of $\theta$ is given by $\cos \theta=\frac{\boldsymbol{u}^{\top} \boldsymbol{w}}{\sqrt{\left(\boldsymbol{u}^{\top} \boldsymbol{w}\right)^{2}+\left(\boldsymbol{u}^{\top} \boldsymbol{w}_{c}\right)^{2}}}$ and $\sin \theta=$ $\frac{\boldsymbol{u}^{\top} \boldsymbol{w}_{c}}{\boldsymbol{w}^{2}+\left(\boldsymbol{u}^{\top} \boldsymbol{w}_{c}\right)^{2}}$. The simplified Procrustes distance is rotation $\sqrt{\left(\boldsymbol{u}^{\top} \boldsymbol{w}\right)^{2}+\left(\boldsymbol{u}^{\top} \boldsymbol{w}_{c}\right)^{2}}$

invariant, ${ }^{1,2}$ and this property is indispensable for some isohedral types because the parameterized tile shape $U$ can only appear in a specific orientation. For example, the two corresponding $J$ edges of the template of IH53 are parameterized such that they are symmetric with respect to the $\mathrm{x}$-axis. This assumption is necessary to express the constraint conditions as linear equations for $\mathrm{IH} 2, \mathrm{IH} 3$, IH5, and IH6 (Figure 3). Henceforth, the simplified Procrustes distance will be referred to as the Procrustes distance, unless otherwise stated.

For other isohedral types (IH1, IH4, IH7, IH21, and IH28), the same result is obtained after the optimization if a simpler distance measure defined as

$$
d_{E}^{2}(U, W)=\sum_{i=1}^{n}\left\|\boldsymbol{u}_{i}-\boldsymbol{w}_{i}\right\|^{2}=\|\boldsymbol{u}-\boldsymbol{w}\|^{2}
$$

is used because the tile shape is parameterized such that it can appear in any orientation [Nagata and Imahori 2020]. We refer to $d_{E}(U, W)$ as the Euclidean distance.

Consider Koizumi and Sugihara's original formulation; i.e., only one configuration $k_{1}=k_{2}=\cdots$ is explored for each isohedral type. If $k_{1}$ is large enough, all possible tile shapes can be approximately generated from the template of each isohedral type, but there is a clear problem. For example, suppose that there exists a tile shape similar to the goal shape and it has a short S edge. When the tile and goal shapes are represented by $n$-point polygons, in order for the two polygons to be evaluated as close using the Euclidean or Procrustes distance, $k_{1}$ points must be densely arranged in the part of the goal polygon that corresponds to the short $S$ edge of the tile polygon. Therefore, the points of the goal polygon $W$ had to be placed manually through trial and error to obtain good results. This problem can be avoided by assigning different numbers of points to the tiling edges and selecting the best configuration of $\left(k_{1}, k_{2}, \ldots\right)$ that yields the minimum distance value, whereas the $n$ points of the goal polygon $W$ are arranged at approximately equal intervals.

Let $I$ be a set of indices for the isohedral types, and $K_{i}(i \in I)$ be defined as a set of all possible configurations of $\left(k_{1}, k_{2}, \ldots\right)$ for the template of IH $i$. For example, $2 k_{1}+k_{2}+k_{3}+k_{4}+k_{5}=n-6$ must hold for IH4. Therefore, $K_{4}=\left\{\left(k_{1}, k_{2}, k_{3}, k_{4}\right) \mid 0 \leq k_{1}, k_{2}, k_{3}, k_{4}, 2 k_{1}+\right.$ $\left.k_{2}+k_{3}+k_{4} \leq n-6\right\}$, where $k_{5}$ is determined by $k_{5}=n-6-$ $\left(2 k_{1}+k_{2}+k_{3}+k_{4}\right)$. As the matrix $B$ depends on $i \in I$ and $k \in K_{i}$, we denote it as $B_{i k}$. In addition, when calculating the Euclidean or Procrustes distance between the two polygons $U$ and $W$, we need to consider $n$ different correspondences by shifting the start point for the numbering of the $n$ points of $U{ }^{3}$ Therefore, the matrix $B_{i k}$ also depends on this numbering scheme. Let $J=\{1,2, \ldots, n\}$ be a set of the indices of the start point for the $n$ different numbering

\footnotetext{
${ }^{1}$ The original Procrustes distance is defined to be scale and rotation invariant.

${ }^{2}$ It is known that the distance value is minimal when $U$ and $W$ have the same centroid.

${ }^{3}$ In previous studies [Nagata and Imahori 2020], $n$ different numbering schemes are considered for $W$, but in this article we use the definition in the text because it is better suited for understanding the proposed method.
}

schemes. For each $j \in J$, we denote the corresponding matrix as $B_{i k j}$, which is obtained from $B_{i k}$ by cyclically shifting down the row elements of $B_{i k}$ by $2(j-1)$.

For each configuration $i \in I, k \in K_{i}$, and $j \in J$, the minimization of the Procrustes distance $d_{P}^{2}(U, W)$ under the constraint $\boldsymbol{u}=B_{i k j} \xi$ is formulated as follows:

$$
\underset{\xi}{\operatorname{argmin}} \xi^{\top} \xi-2 \sqrt{\xi^{\top} B_{i k j}^{\top} V B_{i k j} \xi}+\boldsymbol{w}^{\top} \boldsymbol{w} .
$$

By setting the derivative of the term in Equation (5) equal to zero and solving this equation, the minimum value is given by

$$
\operatorname{dist}_{i k j}^{P}=-\lambda_{i k j}+\boldsymbol{w}^{\top} \boldsymbol{w},
$$

where $\lambda_{i k j}$ is the maximum eigenvalue of $B_{i k j}{ }^{\top} V B_{i k j}$. The optimal solution $\xi_{i k j}^{*}$ is the corresponding eigenvector, whose length is $\sqrt{\lambda_{i k j}}$. The optimal tile shape is then given by $B_{i k j} \xi_{i k j}^{*}$

When the Euclidean distance is applicable, the optimization problem (Equation (5)) can be replaced with

$$
\underset{\xi}{\operatorname{argmin}}\left\|B_{i k j} \xi-w\right\|^{2}
$$

without changing the result. This is a least-squares problem, and the minimum value is given by

$$
\operatorname{dist}_{i k j}^{E}=-\xi_{i k j}^{*}{ }^{\top} \xi_{i k j}^{*}+\boldsymbol{w}^{\top} \boldsymbol{w},
$$

where $\xi_{i k j}^{*}=B_{i k j}{ }^{\top} \boldsymbol{w}$.

The actual EST procedure is described below.

(1) Compute $\operatorname{dist}_{i k j}^{P}$ or dist ${ }_{i k j}^{E}$ (if the Euclidean distance is applicable) and the corresponding tile shapes for all possible configurations of $i \in I, k \in K_{i}$, and $j \in J$. Note that obtained tile shapes occasionally have self-intersection(s) and such tile shapes are discarded.

(2) Display the top $n_{\text {top }}$ tile shapes in terms of the distance value.

Since the tile shape with the minimum distance value is not necessarily the most satisfactory one, multiple tile shapes (e.g., $n_{\text {top }}=10$ ) are displayed so that the user can select the most preferred one.

Nagata and Imahori [2020] developed efficient techniques for solving the optimization problems (Equations (5) and (7)) both in $O(n)$ time. However, the naive EST was still time-consuming because the order of $K_{i}$ is $O\left(n^{3}\right)$ for IH5 and IH6 and $O\left(n^{4}\right)$ for IH4. They further developed an efficient exhaustive search algorithm by introducing an efficient method to calculate lower bounds of $\operatorname{dist}_{i k j}^{E}$ and dist ${ }_{i k j}^{P}$, and the EST can now be performed in a reasonable computation time on a standard modern PC (e.g., $11 \mathrm{~s}$ for $n=120)$.

\section{BASIS OF THE ARAP DEFORMATION}

We outline the basis of the ARAP deformation scheme, mainly according to Sorkine and Alexa [2007]. In a typical surface editing task, given several vertices (handle points) of a mesh representing an object surface and their goal positions specified by the user, the surface is deformed in a physically plausible way as would happen with actual physical objects. In such a deformation, the local structures of the original surface should be preserved as rigidly 


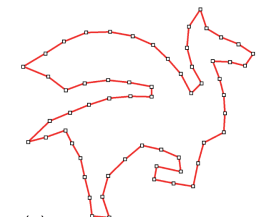

(a)
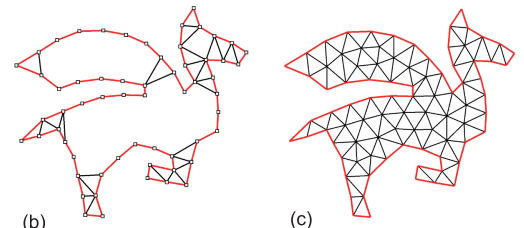

Fig. 5. (a) An example of goal polygon $W$, (b) set $E_{c}$ of the GAD distance $(\gamma=1.6)$, and (c) goal mesh $\tilde{W}$.

as possible on the deformed surface, while distributing distortions uniformly over the surface.

Let a $3 \mathrm{D}$ or $2 \mathrm{D}$ object be represented by a triangular mesh of $n$ vertices, where the positions of the vertices are denoted by $V=\left\{\boldsymbol{v}_{1}, \boldsymbol{v}_{2}, \ldots, \boldsymbol{v}_{n}\right\}$, and a set of vertices connected to vertex $i$ is denoted by $\mathcal{N}(i)$. The positions of the vertices of the deformed mesh are represented by $V^{\prime}=\left\{\boldsymbol{v}_{1}^{\prime}, \boldsymbol{v}_{2}^{\prime}, \ldots, \boldsymbol{v}_{n}^{\prime}\right\}$. The naturalness of the deformed surface is measured using the following deformation energy function:

$$
E\left(V^{\prime}\right)=\sum_{i=1}^{n} \alpha_{i} \sum_{j \in \mathcal{N}(i)} \alpha_{i j}\left\|\left(\boldsymbol{v}_{i}^{\prime}-\boldsymbol{v}_{j}^{\prime}\right)-R_{i}\left(\boldsymbol{v}_{i}-\boldsymbol{v}_{j}\right)\right\|^{2},
$$

where $\alpha_{i}$ and $\alpha_{i j}$ are the cell and edge weights, respectively, and $R_{i}$ represents rotation matrices. For each vertex $i$, the rotation matrix $R_{i}$ is determined to minimize

$$
\sum_{j \in \mathcal{N}(i)} \alpha_{i j}\left\|\left(\boldsymbol{v}_{i}^{\prime}-\boldsymbol{v}_{j}^{\prime}\right)-R_{i}\left(\boldsymbol{v}_{i}-\boldsymbol{v}_{j}\right)\right\|^{2}
$$

which measures the degree of local deformation of a cell defined by vertex $i$ and its neighbors. The goal of the editing task is to find $V^{\prime}$ that minimizes the energy function (Equation (9)) under the constraint that the handle points are fixed to the specified positions.

As we will see in the following sections, the energy function (Equation (9)) can be adapted to the Escherization problem so that it is used instead of the Procrustes and Euclidean distances, where the goal shape is represented as a triangular mesh (Figure 5(c)). However, two technical issues must be addressed.

- In the Escherization problem, only the vertex position of the tile shape boundary can be parameterized. Therefore, we need to model the determination of the positions of the inner points.

- The energy function (Equation (9)) needs to be adapted not only to the Euclidean distance case but also to the Procrustes distance case.

The first issue is similar to a concept known as physics-based volumetric skinning [Hahn et al. 2013] in animation, where the positions of the internal vertices of a volumetric mesh are estimated from the positions of some of the vertices on the surface. Based on the simplicity of the energy function (Equation (9)), this problem can be dealt with straightforwardly.

\section{ESCHERIZATION WITH THE ARAP DEFORMATION}

We introduce a distance function designed based on the ARAP deformation into the EST. We first consider simpler distance functions. The formulations of the EST with these distance functions provide a mathematical basis for the final formulation.

\subsection{Distance Functions Focusing on Local Structures}

We first consider distance functions that measure the similarity between the two polygons $U$ and $W$ based on the "relative position" of the boundary points. When the Euclidean distance is applicable, a distance functions is defined as follows:

$$
d_{G}^{2}(U, W)=\sum_{(i, j) \in E_{c}}\left\|\left(\boldsymbol{u}_{i}-\boldsymbol{u}_{j}\right)-\left(\boldsymbol{w}_{i}-\boldsymbol{w}_{j}\right)\right\|^{2},
$$

where $E_{c}$ is a set of edges on the complete graph induced by the points of $W$. Various distance functions can be designed depending on how set $E_{c}$ is defined, and it is expected that the local structures of the goal polygon $W$ specified by set $E_{c}$ are preserved in the resulting tile polygon $U$.

The simplest form of these distance functions would be obtained when $E_{c}$ is defined as a set of the edges of $W$. We refer to this distance function as the adjacent difference (AD) distance. It is also natural to focus on the similarity of the relative positional relationship of close points. Let $l_{\text {ave }}$ be the average length of the edges of $W$. We define $E_{c}$ as a set of the edges whose lengths are less than $\gamma l_{\text {ave }}$, where the edges of $W$ are always selected and only edges inside the goal polygon $W$ are selected without allowing intersections (shorter edges are preferentially selected). We refer to this distance function as the generalized AD (GAD) distance. Figure 5(b) shows an example of set $E_{c}$ of the GAD distance for a given goal polygon. The appropriate value of $\gamma$ was between 1.2 and 2.0 because larger values weaken the effect of preserving the local structures.

Let $K$ be the Laplacian matrix of the graph defined by $E_{c}$ and the points of $W$. The distance function (Equation (10)) is expressed as in Equation (11) and also as a quadratic form of $\boldsymbol{u}-\boldsymbol{w}$ :

$$
\begin{aligned}
d_{G}^{2}(U, W) & =\sum_{i=1}^{n} \sum_{j=1}^{n}[K]_{i, j}\left(\boldsymbol{u}_{i}-\boldsymbol{w}_{i}\right)^{\top}\left(\boldsymbol{u}_{j}-\boldsymbol{w}_{j}\right) \\
& =(\boldsymbol{u}-\boldsymbol{w})^{\top} G(\boldsymbol{u}-\boldsymbol{w}),
\end{aligned}
$$

where $G\left(\in \mathbb{R}^{2 n \times 2 n}\right)$ is the symmetric matrix whose non-zero elements are given by $[G]_{2 i, 2 j}=[G]_{2 i+1,2 j+1}=[K]_{i, j}(i=$ $1, \ldots, n ; j=1, \ldots, n) .{ }^{4}$ From Equations (1) and (12), the EST with the $(\mathrm{G}) \mathrm{AD}$ distance is performed by solving

$$
\underset{\xi}{\operatorname{argmin}} \xi^{\top} B_{i k j}{ }^{\top} G B_{i k j} \xi-2 \boldsymbol{w}^{\top} G B_{i k j} \xi+\boldsymbol{w}^{\top} G \boldsymbol{w}
$$

for all possible configurations of $i \in I, k \in K_{i}$, and $j \in J$. For each configuration, the solution is obtained by solving the linear equation

$$
B_{i k j}{ }^{\top} G B_{i k j} \xi-B_{i k j}{ }^{\top} G \boldsymbol{w}=0 .
$$

Let $\xi_{i k j}^{*}$ be the solution of this equation. The optimal value is then given by

$$
\operatorname{dist}_{i k j}^{G}=-\xi_{i k j}^{*}{ }^{\top} B_{i k j}{ }^{\top} G B_{i k j} \xi_{i k j}^{*}+\boldsymbol{w}^{\top} G \boldsymbol{w} .
$$

When explicitly distinguishing between the AD and GAD distances, we denote $\operatorname{dist}_{i k j}^{G}$ as dist ${ }_{i k j}^{A D}$ and $\operatorname{dist}_{i k j}^{G A D}$, respectively.

\footnotetext{
${ }^{4}$ Note that 1.0 was further added to $[K]_{1,1}$ to make $B_{i k j}{ }^{\top} G B_{i k j}$ a regular matrix (Equation (14)). Without this, the solution to Equation (14) cannot be uniquely determined because $d_{G}^{2}(U, W)$ is invariant to the translation of the tile shape $U$.
} 
We need to extend Equations (11) to (15) to the Procrustes distance case for some isohedral types. The Procrustes distance version of $d_{G}^{2}(U, W)$ is defined and can be calculated as follows:

$$
d_{G P}^{2}(U, W)=\min _{\theta} \sum_{i=1}^{n} \sum_{j=1}^{n}[K]_{i, j}\left(R(\theta) \boldsymbol{u}_{i}-\boldsymbol{w}_{i}\right)^{\top}\left(R(\theta) \boldsymbol{u}_{j}-\boldsymbol{w}_{j}\right)
$$

$$
=\boldsymbol{u}^{\top} G \boldsymbol{u}-2 \sqrt{\boldsymbol{u}^{\top} G V G \boldsymbol{u}}+\boldsymbol{w}^{\top} G \boldsymbol{w},
$$

where the optimal value of $\theta$ is given by $\cos \theta=$ $\frac{\boldsymbol{u}^{\top} G \boldsymbol{w}}{\sqrt{\left(\boldsymbol{u}^{\top} G \boldsymbol{w}\right)^{2}+\left(\boldsymbol{u}^{\top} G \boldsymbol{w}_{c}\right)^{2}}}$ and $\sin \theta=\frac{\boldsymbol{u}^{\top} G \boldsymbol{w}_{c}}{\sqrt{\left(\boldsymbol{u}^{\top} G \boldsymbol{w}\right)^{2}+\left(\boldsymbol{u}^{\top} G \boldsymbol{w}_{c}\right)^{2}}}$. The derivation of Equation (17) is described in Appendix A.

The minimization of Equation (17) under the constraint $\boldsymbol{u}=$ $B_{i k j} \xi$ is formulated as follows:

$$
\underset{\xi}{\operatorname{argmin}} \xi^{\top} B_{i k j}{ }^{\top} G B_{i k j} \xi-2 \sqrt{\xi^{\top} B_{i k j}{ }^{\top} G V G B_{i k j} \xi}+\boldsymbol{w}^{\top} G \boldsymbol{w} .
$$

The solution $\xi_{i k j}^{*}$ and the optimal value $\operatorname{dist}_{i k j}^{G P}$ of this optimization problem are obtained by solving the generalized eigenvalue problem:

$$
B_{i k j}{ }^{\top} G V G B_{i k j} \xi=\lambda B_{i k j}{ }^{\top} G B_{i k j} \xi .
$$

Let $\lambda_{i k j}$ be the maximum eigenvalue of this generalized eigenvalue problem. The solution $\xi_{i k j}^{*}$ is given by the corresponding eigenvector whose length is determined to satisfy $\xi_{i k j}^{*}{ }^{\top} B_{i k j}{ }^{\top} G B_{i k j} \xi_{i k j}^{*}=$ $\lambda_{i k j}$. The optimal value is then given by

$$
\operatorname{dist}_{i k j}^{G P}=-\lambda_{i k j}+\boldsymbol{w}^{\top} G \boldsymbol{w} .
$$

The derivation of Equations (19) and (20) is presented in Appen$\operatorname{dix}$ A.

It takes $O\left(n^{3}\right)$ time to solve the optimization problem (Equation (13)) using the Cholesky decomposition of the matrix $B_{i k j}{ }^{\top} G B_{i k j}$ for solving Equation (14). The optimization problem (Equation (18)) can be solved with the same time complexity. In general, for a symmetric matrix $A\left(=B_{i k j}{ }^{\top} G V G B_{i k j}\right)$ and a symmetric positive-definite matrix $B\left(=B_{i k j}{ }^{\top} G B_{i k j}\right)$, the generalized eigenvalue problem $A x=\lambda B x$ can be converted into an eigenvalue problem $\left(L^{-1} A\left(L^{-1}\right)^{\top}\right) \boldsymbol{y}=\lambda \boldsymbol{y}$, where $\boldsymbol{y}=L^{\top} \boldsymbol{x}$ and $L$ is a lower triangular matrix such that $B=L L^{\top}$ (the Cholesky decomposition). This technique was used to solve the generalized eigenvalue problem (Equation (19)). Once it is converted into the eigenvalue problem, the maximum eigenvalue and corresponding eigenvector can be computed in $O(n)$ time, as in the case of the Procrustes distance [Nagata and Imahori 2020].

\subsection{Distance Function Considering the Internal Shape}

Next, we represent the goal and tile shapes as meshes, where the similarity between the two shapes is evaluated by considering the local structures near the boundary and inside the shapes. The rotation matrices $R_{i}$ (Equation (9)) are not introduced here.

Let the goal shape be represented as a triangular mesh formed from the original simple closed polygon $W$ by adding inner points and links. Figure 5(c) shows an example of the mesh representation of a goal shape. The tile shape is also represented as a triangular mesh with the same connectivity structure. We denote by $\tilde{W}$ and $\tilde{U}$ the meshes representing the goal and tile shapes, respectively.
For a given goal polygon $W$, the mesh $\tilde{W}$ was created such that the lengths of the inner edges are roughly equal to the average length of the edges of $W$. We constructed the mesh $\tilde{W}$ using a local search-based method, where the positions of the inner points were iteratively perturbed while adding or deleting edges in the internal area, starting from roughly determined initial positions. Details of this procedure are omitted because the tiling results did not depend on small differences in the mesh $\tilde{W}$.

Let $n^{\prime}$ be the number of the inner points of the mesh $\tilde{W}(\tilde{U})$, where the inner points are numbered from $n+1$ to $n+n^{\prime}$, and the $n$ points on the boundary are numbered in the same way as for $W$ $(U)$. We denote the coordinates of $\tilde{U}$ and $\tilde{W}$ by

$$
\tilde{\boldsymbol{u}}=\left(\begin{array}{c}
\boldsymbol{u} \\
\boldsymbol{u}^{\prime}
\end{array}\right) \text { and } \tilde{\boldsymbol{w}}=\left(\begin{array}{c}
\boldsymbol{w} \\
\boldsymbol{w}^{\prime}
\end{array}\right)
$$

respectively, where $\boldsymbol{u}^{\prime}$ and $\boldsymbol{w}^{\prime}$ are the coordinates of the inner points. The coordinates of the $i$ th point of $\tilde{U}$ and $\tilde{W}$ are represented as $\boldsymbol{u}_{i}$ and $\boldsymbol{w}_{i}$, respectively. Let $\mathcal{N}(i)$ be a set of the points of $\tilde{W}(\tilde{U})$ connected to point $i$. A distance function between $\tilde{U}$ and $\tilde{W}$ is then defined and represented as follows:

$$
\begin{aligned}
& d_{I}^{2}(\tilde{U}, \tilde{W})=\frac{1}{2} \sum_{i=1}^{n+n^{\prime}} \alpha_{i} \sum_{j \in \mathcal{N}(i)}\left\|\left(\boldsymbol{u}_{i}-\boldsymbol{u}_{j}\right)-\left(\boldsymbol{w}_{i}-\boldsymbol{w}_{j}\right)\right\|^{2} \\
& =(\tilde{\boldsymbol{u}}-\tilde{\boldsymbol{w}})^{\top} \tilde{G}(\tilde{\boldsymbol{u}}-\tilde{\boldsymbol{w}}) \\
& =(\boldsymbol{u}-\boldsymbol{w})^{\top} G_{0}(\boldsymbol{u}-\boldsymbol{w})+2(\boldsymbol{u}-\boldsymbol{w})^{\top} G_{1}\left(\boldsymbol{u}^{\prime}-\boldsymbol{w}^{\prime}\right) \\
& \quad+\left(\boldsymbol{u}^{\prime}-\boldsymbol{w}^{\prime}\right)^{\top} G_{2}\left(\boldsymbol{u}^{\prime}-\boldsymbol{w}^{\prime}\right) .
\end{aligned}
$$

Note that we do not consider edge weights $\alpha_{i j}$ used in Equation (9) for simplicity. If the weights $\alpha_{i}$ are all 1 , the matrix $\tilde{G}\left(\in \mathbb{R}^{2\left(n+n^{\prime}\right) \times 2\left(n+n^{\prime}\right)}\right)$ is defined from the Laplacian matrix of the mesh $\tilde{W}$ in the same way as when $G$ is constructed from $K$ (Equation (12)). In the weighted setting, the Laplacian matrix is modified accordingly. In the last expression (Equation (24)), the distance function is represented in a quadratic form of $\boldsymbol{u}^{\prime}-\boldsymbol{w}^{\prime}$, where $G_{0}\left(\in \mathbb{R}^{2 n \times 2 n}\right)$ is the top-left corner of $\tilde{G}, G_{1}\left(\in \mathbb{R}^{2 n \times 2 n^{\prime}}\right)$ is the top-right corner of $\tilde{G}$, and $G_{2}\left(\in \mathbb{R}^{2 n^{\prime} \times 2 n^{\prime}}\right)$ is the bottom-right corner of $\tilde{G}$. The matrices $\tilde{G}, G_{0}$, and $G_{2}$ are symmetric.

Since only the positions of the points on the boundary of $\tilde{U}$ are parameterized, we want to remove $\boldsymbol{u}^{\prime}$ from the distance function. Therefore, the positions of the inner points of $\tilde{U}$ are determined to minimize $d_{I}^{2}(\tilde{U}, \tilde{W})$. By solving $\frac{\partial d_{I}^{2}(\tilde{U}, \tilde{W})}{\partial \boldsymbol{u}^{\prime}}=0$, we have

$$
\boldsymbol{u}^{\prime}-\boldsymbol{w}^{\prime}=-G_{2}^{-1} G_{1}^{\top}(\boldsymbol{u}-\boldsymbol{w}) .
$$

By substituting Equation (25) into Equation (24), we finally obtain the following energy function with respect to $\boldsymbol{u}$ :

$$
\begin{aligned}
E_{I}(\boldsymbol{u}) & =\min _{\boldsymbol{u}^{\prime}} d_{I}^{2}(\tilde{U}, \tilde{W}) \\
& =(\boldsymbol{u}-\boldsymbol{w})^{\top}\left(G_{0}-G_{1} G_{2}^{-1} G_{1}^{\top}\right)(\boldsymbol{u}-\boldsymbol{w}) .
\end{aligned}
$$

We may refer to this energy function as the $E_{I}$ distance in the context of the Escherization problem. If we define the symmetric matrix $G_{I}=G_{0}-G_{1} G_{2}{ }^{-1} G_{1}^{\top}$, which is computed only once at the beginning of the EST, the energy function $E_{I}(\boldsymbol{u})$ has the same form as the distance function $d_{G}^{2}(U, W)$ (Equation (12)). Therefore, for each configuration $(i, k, j)$, the minimum value of $E_{I}(\boldsymbol{u})$ under the constraint $\boldsymbol{u}=B_{i k j} \xi$ and the optimal tile shape are obtained in the 
same way as in Equations (13) to (15) by simply replacing $G$ with $G_{I}$. Consequently, the minimum value of $E_{I}(\boldsymbol{u})$ is given by

$$
\operatorname{dist}_{i k j}^{I}=-\xi_{i k j}^{*}{ }^{\top} B_{i k j}{ }^{\top} G_{I} B_{i k j} \xi_{i k j}^{*}+\boldsymbol{w}^{\top} G_{I} \boldsymbol{w},
$$

where $\xi_{i k j}^{*}$ is the solution of the linear equation $B_{i k j}{ }^{\top} G_{I} B_{i k j} \xi-$ $B_{i k j}{ }^{\top} G_{I} \boldsymbol{w}=0$. We can see that the value of dist ${ }_{i k j}^{I}$ does not depend on the positions of the inner points of $\tilde{W}$ (depends on the connectivity structure of $\tilde{W}$ ), although it seems strange at first glance.

The energy function $E_{I}(\boldsymbol{u})$ can be extended to its Procrustes distance version $E_{I P}(\boldsymbol{u})$. Let $R_{n}(\theta)\left(\in \mathbb{R}^{2 n \times 2 n}\right)$ be the matrix that rotates $n$ points by an angle $\theta$. The energy function $E_{I P}(\boldsymbol{u})$ is defined by $\min _{\theta} E_{I}\left(R_{n}(\theta) \boldsymbol{u}\right)$. Similar to the derivation of $E_{I}(\boldsymbol{u}), E_{I P}(\boldsymbol{u})$ has the same form as the distance function $d_{G P}^{2}(U, W)$ (Equation (17)), with $G$ being replaced with $G_{I}$. The derivation is given in Appendix B. For each configuration $(i, k, j)$, the minimum value of $E_{I P}(\boldsymbol{u})$ and the optimal tile shape are obtained in the same way as in Equations (18) to (20) by simply replacing $G$ with $G_{I}$. Consequently, we obtain the shape of the boundary of the optimal tile shape. The corresponding mesh representation can be obtained as follows. First, the boundary shape is rotated by the optimal angle $\theta$ (Equation (17)). Then, the position of the inner points are calculated from the rotated boundary shape using Equation (25).

\subsection{Distance Function Based on ARAP Deformation}

We now consider a distance function based on the ARAP deformation scheme.

4.3.1 Formulation. For each point $i$ of the mesh $\tilde{W}(\tilde{U})$, let a cell $C_{i}\left(C_{i}^{\prime}\right)$ be defined by the edges between point $i$ and its neighboring points $j \in \mathcal{N}(i)$. The distance function is then defined as follows:

$$
d_{I R}^{2}(\tilde{U}, \tilde{W})=\frac{1}{2} \sum_{i=1}^{n+n^{\prime}} \alpha_{i} \sum_{j \in \mathcal{N}(i)}\left\|\left(\boldsymbol{u}_{i}-\boldsymbol{u}_{j}\right)-R_{i}\left(\boldsymbol{w}_{i}-\boldsymbol{w}_{j}\right)\right\|^{2},
$$

where $R_{i}$ denotes the rotation matrix. For each point $i$, the rotation matrix $R_{i}$ is determined to minimize

$$
\sum_{j \in \mathcal{N}(i)}\left\|\left(\boldsymbol{u}_{i}-\boldsymbol{u}_{j}\right)-R_{i}\left(\boldsymbol{w}_{i}-\boldsymbol{w}_{j}\right)\right\|^{2},
$$

which measures the degree of local deformation from $C_{i}$ to $C_{i}^{\prime}$. In contrast to the $3 \mathrm{D}$ case in which the singular value decomposition of a matrix is required [Sorkine and Alexa 2007], in the 2D case, the matrix $R_{i}$ can be calculated more efficiently, as described later.

The rotation matrices $R_{i}\left(1 \leq i \leq n+n^{\prime}\right)$ depend on $\tilde{\boldsymbol{u}}$, and therefore, the distance function $d_{I R}^{2}(\tilde{U}, \tilde{W})$ cannot be written as a quadratic function of $\tilde{\boldsymbol{u}}$. For this reason, a closed-form solution for minimizing $d_{I R}^{2}(\tilde{U}, \tilde{W})$ under the constraint $\boldsymbol{u}=B_{i k j} \xi$ does not exist. Instead, we will minimize $d_{I R}^{2}(\tilde{U}, \tilde{W})$ via a two-step closedform iterative procedure in the same way as in Sorkine and Alexa [2007]: (1) the minimization of $d_{I R}^{2}(\tilde{U}, \tilde{W})$ with the rotation matrices $R_{i}$ being fixed and (2) the estimation of the rotation matrices $R_{i}$ with the positions of $\tilde{U}$ being fixed are alternatively performed.

4.3.2 Iterative Procedure. Let the rotation matrices $R_{i}(1 \leq i \leq$ $\left.n+n^{\prime}\right)$ be initialized to the identity matrix. The first step is performed as follows. If the rotation matrices $R_{i}$ are fixed, the distance function $d_{I R}^{2}(\tilde{U}, \tilde{W})$ can be written as a quadratic form of $\tilde{\boldsymbol{u}}$ :

$$
d_{I R}^{2}(\tilde{U}, \tilde{W})=\tilde{\boldsymbol{u}}^{\top} \tilde{G} \tilde{\boldsymbol{u}}-2 \tilde{\boldsymbol{u}}^{\top} \tilde{T} \tilde{\boldsymbol{w}}+\tilde{\boldsymbol{w}}^{\top} \tilde{G} \tilde{\boldsymbol{w}},
$$

where $\tilde{T}\left(\in \mathbb{R}^{2\left(n+n^{\prime}\right) \times 2\left(n+n^{\prime}\right)}\right)$ is a matrix. Let $\tilde{T} \cdot \operatorname{block}(i, j, 2,2)$ represent the $2 \times 2$ block matrix of $\tilde{T}$ starting at position $(i, j)$. The matrix $\tilde{T}$ is computed as follows:

$$
\begin{aligned}
& \tilde{T}=\mathbf{0} \\
& \text { for } i=1, \ldots, n+n^{\prime} \\
& \quad \text { for } j \in \mathcal{N}(i) \\
& \tilde{T} \cdot \operatorname{block}(2 i, 2 i, 2,2)+=\alpha_{i} R_{i} \\
& \tilde{T} \cdot \operatorname{block}(2 j, 2 j, 2,2)+=\alpha_{i} R_{i} \\
& \tilde{T} \cdot \operatorname{block}(2 i, 2 j, 2,2)-=\alpha_{i} R_{i} \\
& \tilde{T} \cdot \operatorname{block}(2 j, 2 i, 2,2)-=\alpha_{i} R_{i}
\end{aligned}
$$

As in Equation (27), we need to remove $\boldsymbol{u}^{\prime}$ from the distance function by determining the positions of the inner points such that $d_{I R}^{2}(\tilde{U}, \tilde{W})$ is minimized. Let $T\left(\in \mathbb{R}^{2 n \times 2\left(n+n^{\prime}\right)}\right)$ and $T^{\prime}(\in$ $\left.\mathbb{R}^{2 n^{\prime} \times 2\left(n+n^{\prime}\right)}\right)$ be the first $2 n$ rows and the last $2 n^{\prime}$ rows of $\tilde{T}$, respectively. Then, $d_{I R}^{2}(\tilde{U}, \tilde{W})$ can be written as a quadratic form of $u^{\prime}$ :

$$
\begin{aligned}
d_{I R}^{2}(\tilde{U}, \tilde{W})= & \boldsymbol{u}^{\top} G_{0} \boldsymbol{u}+2 \boldsymbol{u}^{\top} G_{1} \boldsymbol{u}^{\prime}+\boldsymbol{u}^{\prime \top} G_{2} \boldsymbol{u}^{\prime} \\
& -2\left(\boldsymbol{u}^{\top} T \tilde{\boldsymbol{w}}+\boldsymbol{u}^{\prime \top} T^{\prime} \tilde{\boldsymbol{w}}\right)+\tilde{\boldsymbol{w}}^{\top} \tilde{G} \tilde{\boldsymbol{w}} .
\end{aligned}
$$

By solving $\frac{\partial d_{I R}^{2}(\tilde{U}, \tilde{W})}{\partial \boldsymbol{u}^{\prime}}=0$, we have the positions of the inner points:

$$
\boldsymbol{u}^{\prime}=G_{2}^{-1}\left(T^{\prime} \tilde{\boldsymbol{w}}-G_{1}^{\top} \boldsymbol{u}\right) .
$$

By substituting Equation (33) into Equation (32), we finally obtain the following energy function with respect to $\boldsymbol{u}$ :

$$
\begin{aligned}
& E_{I R}(\boldsymbol{u})=\min _{\boldsymbol{u}^{\prime}} d_{I R}^{2}(\tilde{U}, \tilde{W}) \\
& =\boldsymbol{u}^{\top}\left(G_{0}-G_{1} G_{2}{ }^{-1} G_{1}^{\top}\right) \boldsymbol{u}-2 \tilde{\boldsymbol{w}}^{\top}\left(T^{\top}-T^{\prime \top} G_{2}{ }^{-1} G_{1}{ }^{\top}\right) \boldsymbol{u} \\
& \quad+\tilde{\boldsymbol{w}}^{\top}\left(\tilde{G}-T^{\prime \top} G_{2}{ }^{-1} T^{\prime}\right) \tilde{\boldsymbol{w}} .
\end{aligned}
$$

We may refer to this energy function as the $E_{I R}$ distance in the context of the Escherization problem.

Recall that for each configuration $(i, k, j)$, the boundary of the tile shape $\tilde{U}$ is parameterized by $\boldsymbol{u}=B_{i k j} \xi$, and therefore, the energy function $E_{I R}(\boldsymbol{u})$ can be expressed as a quadratic function of $\xi$. The optimal tile shape is obtained by solving $\frac{\partial E_{I R}(\boldsymbol{u})}{\partial \xi}=0$, which is calculated as follows:

$$
B_{i k j}{ }^{\top} G_{I} B_{i k j} \xi=B_{i k j}{ }^{\top}\left(T-G_{1} G_{2}^{-1} T^{\prime}\right) \tilde{\boldsymbol{w}},
$$

where $G_{I}=G_{0}-G_{1} G_{2}{ }^{-1} G_{1}^{\top}$. Let $\xi_{i k j}^{*}$ be the solution of this equation. The positions of the points on the boundary of the optimal tile shape are given by $\boldsymbol{u}^{*}=B_{i k j} \xi_{i k j}^{*}$, and the positions of the inner points are obtained from Equation (33) if necessary. The minimum value of $E_{I R}(\boldsymbol{u})$ under the constraint $\boldsymbol{u}=B_{i k j} \xi$ is then given by

$$
\operatorname{dist}_{i k j}^{I R}=-\xi_{i k j}^{*}{ }^{\top} B_{i k j}{ }^{\top} G_{I} B_{i k j} \xi_{i k j}^{*}+\tilde{\boldsymbol{w}}^{\top}\left(\tilde{G}-T^{\prime \top} G_{2}{ }^{-1} T^{\prime}\right) \tilde{\boldsymbol{w}} .
$$

Given the tile shape $\tilde{U}$ determined in the first step, the second step is performed by calculating the matrix $R_{i}$ that minimizes Equation (30) for every $i\left(1 \leq i \leq n+n^{\prime}\right)$. Let $\boldsymbol{w}_{i}-\boldsymbol{w}_{j}$ and $\boldsymbol{u}_{i}-\boldsymbol{u}_{j}$ be denoted as $\boldsymbol{e}$ and $\boldsymbol{e}^{\prime}$, respectively. Equation (30) is then written as $\sum_{j \in \mathcal{N}(i)}\left\|R_{i} \boldsymbol{e}_{i j}-\boldsymbol{e}^{\prime}{ }_{i j}\right\|^{2}$. Therefore, the rotation matrix $R_{i}$ 


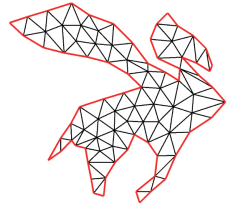

(a) iteration $=1$

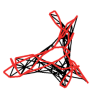

(b) iteration $=1$

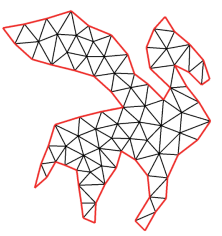

iteration $=3$

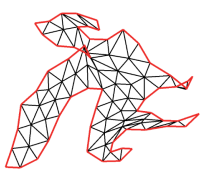

iteration $=5$

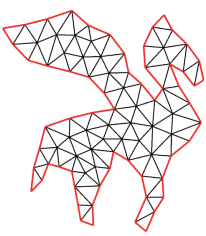

iteration $=20$

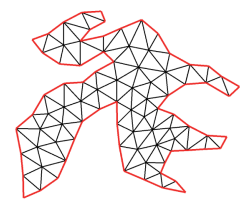

iteration $=10$
Fig. 6. Changes in the tile shape during the iterative procedure using the energy functions (a) $E_{I R P}(\boldsymbol{u})$ and (b) $E_{I R}(\boldsymbol{u})$. In the former case, the displayed tile shapes $\tilde{U}$ are rotated so that their orientation matches that of the goal shape, but the parameterized tile shapes are oriented as in the latter case. The Procrustes distance must be used for the isohedral type (IH6) used in this example.

that minimizes this function can be obtained as the optimal angle $\theta$ that minimizes the simplified Procrustes distance (Equation (2)) between the two point $\operatorname{sets}^{5}\left\{\boldsymbol{e}_{i 1}, \boldsymbol{e}_{i 2}, \ldots\right\}$, and $\left\{\boldsymbol{e}_{i 1}^{\prime}, \boldsymbol{e}_{i 2}^{\prime}, \ldots\right\}$.

The first and second steps are iterated until the value of $\operatorname{dist}_{i k j}^{I R}$ converges. In the experimental setup, the number of iterations was set to three based on the trade-off between the degree of convergence and the computation time. After the matrices $R_{i}$ are computed at the second step of the final iteration, the value of $\operatorname{dist}_{i k j}^{I R}$ is re-computed using the new value of the matrix $\tilde{T}$, and the resulting value is used to evaluate the tile shape determined at the first step of the final iteration.

This iterative procedure is not guaranteed to yield a global optimal solution but always converges [Sorkine and Alexa 2007] if iterations were repeated enough times. Figure 6(a) shows a typical example of the tile shapes $\tilde{U}$ obtained during the iterative procedure for the goal mesh $\tilde{W}$ presented in Figure 5, where the caption of Figure 6 should be ignored here. We can see that the tile shape is almost converged at the iteration count of three. One may think that the convergence point depends on the initial guess of the rotation matrices $R_{i}$. In preliminary experiments, the iterative procedure was tested starting from random rotation matrices $R_{i}$ for each $i$. The iterative procedure always converged on almost the same tile shape as when the matrices $R_{i}$ were initialized to the identity matrix, although the number of iterations to convergence was increased.

4.3.3 Procrustes Distance Case. The energy function $E_{I R}(\boldsymbol{u})$ can be extended to its Procrustes distance version $E_{I R P}(\boldsymbol{u})$. Using the matrix $R_{n}(\theta)$ (see Section 4.2), the energy function $E_{I R P}(\boldsymbol{u})$ is defined by $\min _{\theta} E_{I R}\left(R_{n}(\theta) \boldsymbol{u}\right)$. Let $\boldsymbol{u}^{*}$ be the solution that minimizes $E_{I R P}(\boldsymbol{u})$ in the first step of the iterative procedure. In the second step, the matrices $R_{i}$ are computed for the tile mesh $\tilde{U}$ rotated by an angle $\theta^{*}=\operatorname{argmin}_{\theta} E_{I R}\left(R_{n}(\theta) \boldsymbol{u}^{*}\right)$ (Figure 6(a)). That

${ }^{5}$ It may be better to translate one set so that the two sets have the same centroid, but this was not done because there was no particular change in the results. is, the positions of the boundary points are given by $R_{n}\left(\theta^{*}\right) \boldsymbol{u}^{*}$, and the positions of the inner points are obtained by setting $\boldsymbol{u}=R_{n}\left(\theta^{*}\right) \boldsymbol{u}^{*}$ in Equation (33). Details of the energy function $E_{I R P}(\boldsymbol{u})$ are described in Appendix B.

From the concept of ARAP deformation (Equations (29) and (30)), the energy function $E_{I R}(\boldsymbol{u})$ may be applicable as it is for the isohedral types to which the Procrustes distance must be used because each of the rotation matrices $R_{i}$ is locally optimized. In the preliminary experiments, we confirmed that the same result was always obtained using either $E_{I R}(\boldsymbol{u})$ or $E_{I R P}(\boldsymbol{u})$ if the number of iterations was sufficiently large. As expected, the iterative procedure converged with a lower number of iterations when $E_{I R P}(\boldsymbol{u})$ was used, and therefore, this energy function was employed.

Figure 6 shows examples of the tile shapes $\tilde{U}$ obtained during the iterative procedure using the energy functions $E_{I R P}(\boldsymbol{u})$ and $E_{I R}(\boldsymbol{u})$ for the goal mesh $\tilde{W}$ presented in Figure 5. For the isohedral type used in this example, the Procrustes distance must be used. When $E_{I R P}(\boldsymbol{u})$ is used, the tile shape is almost converged at the third iteration. Conversely, when $E_{I R}(\boldsymbol{u})$ is used, the tile shape does not converge at the 5 th iteration, but it eventually converges to the same shape as the former at around the 10th iteration.

\section{HEURISTIC SEARCH}

The EST with each of the GAD, $E_{I}$, and $E_{I R}$ distance is, however, time-consuming, and we develop an alternative approach to obtain results in a reasonable computation time. We refer to this approach as heuristic search. The explanation is given for the case where the Euclidean distance is available, but the same approach is applicable for the Procrustes distance case.

The basic idea is to compute the value of dist $_{i k j}^{G A D}$ (Equation (15)), dist ${ }_{i k j}^{I}$ (Equation (28)), or dist ${ }_{i k j}^{I R}$ (Equation (37)) for only promising configurations of $(i, k, j)$. To select promising configurations, we utilize the results of the EST with the AD distance, which can be obtained in a relatively short computation. Assuming that the top-ranked configurations of $(i, k, j)$ in the EST with each of the $\mathrm{GAD}, E_{I}$, and $E_{I R}$ distance are correlated with those of the AD distance, it is rational to search for only configurations of $(i, k, j)$ that are high-ranked in the EST with the AD distance.

The actual heuristic search procedure with the $E_{I R}$ distance is as follows:

(1) Perform the EST with the AD distance and store the top $n_{A D}$ configurations of $(i, k, j)$. Note that unlike the original EST, tile shapes including self-intersection are not discarded.

(2) For all the selected $n_{A D}$ configurations of $(i, k, j)$, compute $\operatorname{dist}_{i k j}^{I R}$ and the corresponding tile shapes.

(3) Display the top $n_{\text {top }}$ tile shapes in terms of the distance value.

When using the GAD or $E_{I}$ distance in the heuristic search, dist ${ }_{i k j}^{I R}$ is replaced with dist ${ }_{i k j}^{G A D}$ or dist ${ }_{i k j}^{I}$ in the above procedure.

The parameter $n_{A D}$ may need to be changed depending on the value of $n$. For example, the number of all possible configurations of $(i, k, j)$ is $7.9 \times 10^{6}$ and $1.3 \times 10^{8}$ for $n=60$ and 120 , respectively. For simplicity, $n_{A D}$ was set to 10,000 for the GAD and $E_{I}$ distances and 50,000 for the $E_{I R}$ distance, regardless of the value of $n$. Some of the top $n_{\text {top }}$ solutions of the EST may be overlooked in 
the heuristic search, but most of them were obtained as described in Section 6. One may think that the Euclidean distance can be used in step (1), but the majority of the top $n_{\text {top }}$ solutions were lost in that setting.

Although the EST with the AD distance and the EST with the GAD distance are formulated as the same optimization problem (Equation (13)), the former can be performed much faster. The basic idea is to transform the optimization problem (Equation (13)) for the $\mathrm{AD}$ distance into the form of the optimization problem (Equation (7)) for the Euclidean distance, which makes it possible to utilize a technique developed to speed up the EST with the Euclidean distance (see the end of Section 2.2). Using this technique, a lower bound of dist ${ }_{i k j}^{A D}$ can be efficiently computed, and the computation of dist ${ }_{i k j}^{A D}$ is skipped if there is no possibility of updating the current top $n_{A D}$ solutions. See Nagata and Imahori [2020] for details on the computation of the lower bound of $\operatorname{dist}_{i k j}^{E}$. Appendix $C$ describes how the optimization problem (Equation (13)) for the $\mathrm{AD}$ distance is transformed into a form of the optimization problem (Equation (7)).

\section{EXPERIMENTAL RESULTS}

\subsection{Experimental Setting}

The EST was performed using the Euclidean, $\mathrm{AD}, \mathrm{GAD}, E_{I}$, and $E_{I R}$ distances. For the GAD, $E_{I}$, and $E_{I R}$ distances, the heuristic search was also performed. All algorithms were applied to six goal meshes $\tilde{W}$ or goal polygons $W$ shown in Figure 8, where the corresponding goal figures are also illustrated. All the algorithms were implemented in $\mathrm{C}++$ with the Eigen library $(\mathrm{C}++$ library for linear algebra) on Ubuntu 16.04 Linux. The programs were executed on PCs, each with an Intel Core i9-10900K 3.7-GHz CPU. The source code is available at https://github.com/nagata-yuichi/EscherTiling.

For both the EST and the heuristic search, $n_{\text {top }}$ was set to 10 . For the $E_{I}$ and $E_{I R}$ distances, the parameter $\alpha_{i}$ was set to $1.0(1 \leq$ $i \leq n)$ and $0.5\left(n+1 \leq i \leq n+n^{\prime}\right)$. This parameter setting was motivated to emphasize the local structure of the boundary. For the GAD distance, the parameter $\gamma$ was set to $1.4,1.6$, and 2.0, and an appropriate parameter value was selected; the selected values are 1.4 for goal shapes squid, spider, and deer and 1.6 for the others. The sets $E_{c}$ of the GAD distance are shown in Figure 8.

Tile figures presented in this section were generated by deforming the goal figures based on the deformation of the cells between the goal mesh $\tilde{W}$ and the tile mesh $\tilde{U}$. Note that tile shapes are originally represented as simple closed polygons for the Euclidean, $A D$, and GAD distances. Therefore, these were converted to meshes having the same structures as those defined for the $E_{I}$ distance, where the positions of the inner points were determined according to Equation (25).

For each distance function on each goal figure, one or two most satisfactory tile figures were determined by questionnaire among the top 10 tile figures obtained by the EST, and the selected ones are mainly presented. In the questionnaire, very similar tile figures were excluded from the top 10 tile figures beforehand, and 13 people voted for one tile shape that they thought was obtained with the most natural deformation. The first and second places were then determined according to the number of votes received. All

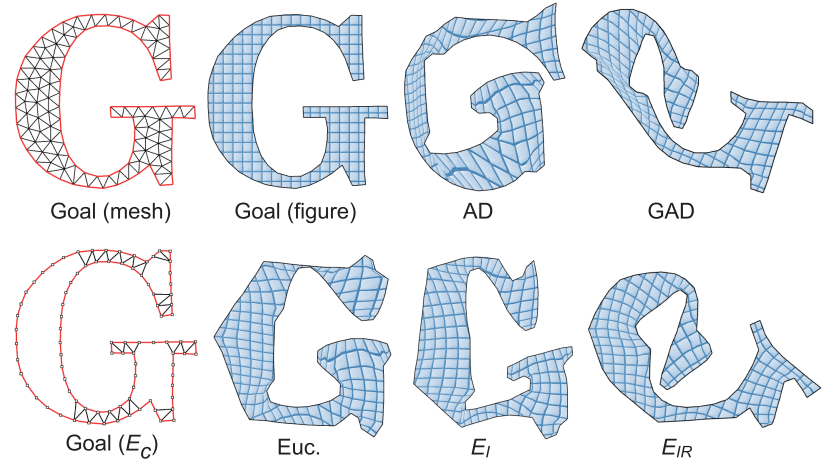

Fig. 7. Tile shapes obtained by the EST with the five distance functions for goal figure "G," which is regarded as an elastic object rather than a letter.

the top 10 tile figures are presented in the online supplementary file.

\subsection{Preliminary Results}

Before proceeding to the results for the six goal figures, we verify the effects of the five distance functions on the results for a goal figure with easy-to-understand features. Figure 7 shows the results of the EST with the five distance functions on a goal figure " $G$ " $\left(n=88, n^{\prime}=40\right)$. This goal figure has locally detailed structures at both ends and a curve with a continuously varying width in the middle. Note that our motivation here is not to generate a tile shape that looks like a letter $G$, but to generate a tile shape that is obtained by a natural deformation of the goal shape when it is regarded as an elastic object. For this purpose, it is important to maintain the detailed structures of the goal shape in the obtained tile shape. It is also important to maintain the smoothness of the smooth part of the boundary of the goal shape. For each distance function, at most two types of tile shapes were obtained, and a typical one is displayed in the figure.

The main problems with the tile shape obtained with the Euclidean distance are that the detailed structure in the upper right corner is broken, the upper part is squashed, and some originally smooth boundary parts become angular. Compared to this tile shape, the shape of the boundary is better preserved in the tile shape obtained with the $\mathrm{AD}$ distance, but a significant volume change is observed inside the shape. The detailed structures at both ends are further preserved in the tile shape obtained with the GAD distance. This is a natural consequence because many of the edges of set $E_{c}$ are concentrated in these areas. However, the local shapes of the other parts are heavily distorted.

As expected from the concept of the $E_{I}$ distance, the local structures are well maintained throughout the tile shape obtained with this distance function. Therefore, a significant volume change, as in the tile shapes obtained with the $\mathrm{AD}$ and GAD distances, is avoided. However, deformations are locally concentrated in a few areas, and the boundary of the obtained tile shape is unnecessarily ragged. On the other hand, such excessive local deformations are suppressed in the tile shape obtained with the $E_{I R}$ distance by distributing distortions more uniformly. This may result in a large deformation, but such a deformation is physically plausible as long as the local structures are not significantly distorted. 
The effect of the $E_{I R}$ distance described above is predictable from the concept of ARAP deformation, but it is interesting to find appropriate global deformations that cannot be predicted in advance. Meanwhile, in a typical shape editing task, the purpose of using ARAP deformation is to generate a deformed shape of an object that is expected from a deformation operation given to the object.

\subsection{Main Results}

We move on to the results for the six goal figures (Figure 8). First, the results of the Euclidean and AD distances are summarized. Figure 9 shows the results of the EST with these distance functions where the most satisfactory tile figures selected by the questionnaire are presented. The results indicate that the displayed tile figures contain noticeable unnatural deformations, e.g., large changes in the thickness of limbs and large volume changes of the body parts. This indicates that it is difficult to obtain satisfactory tile shapes using the Euclidean distance when the goal shapes are complex. The same can be said for the AD distance, but the AD distance is necessary in the heuristic search.

Figure 10 shows the results of the EST with the GAD, $E_{I}$, and $E_{I R}$ distances where the two most satisfactory tile figures selected by the questionnaire are presented. It can be observed that the use of the GAD distance reduces the unnatural deformations that occur in the Euclidean and AD distance cases. This is an expected result because the detailed structures specified by the sets $E_{c}$ would be better preserved in the obtained tile shapes. In fact, some of the top 10 tile shapes obtained with the GAD distance involve significant volume changes (see the online supplementary file), and whether the internal shape not specified by the set $E_{c}$ is well preserved depends on chance.

Due to the expected effects of the GAD, $E_{I}$, and $E_{I R}$ distances, the resulting tile figures are expected to be more satisfactory in this order. However, for each goal figure, it was difficult to determine which of the tile figures shown in Figure 10 was the most satisfactory when these had different global structures. For example, if tile figures $E_{I}(5)$ and $E_{I R}(1)$ of spider are compared, which one is better strongly depends on personal preference. On the other hand, if tile figures have a similar global structure, we can see the expected differences between them. Tile figures generated with the three distance functions under the same or similar configuration of $(i, k, j)$ tend to be similar, and such tile figures are linked by the solid or dotted lines in Figure 10. For example, if tile figures GAD(7) and $E_{I}(2)$ of deer are compared, we can see that the volume of the body part and the shape of the jaw are better maintained in the latter. Similarly, when comparing two tile figures $E_{I}(1)$ and $E_{I R}(3)$ of deer, the latter has sharper antlers, legs, and tail. Similar improvements can be seen for pairs of tile figures joined by other links.

If the user prefers a tile figure obtained with the $E_{I}$ or GAD distance, there is a possibility that this tile figure can be improved using the $E_{I R}$ distance. For example, if tile figure $E_{I}(2)$ of deer is selected, we can optimize the tile shape using the $E_{I R}$ distance under the same configuration of $(i, k, j)$ that generates $E_{I}(2)$. The obtained tile figure is displayed in Figure 11; we can see that the sharpness of the antlers and legs is improved. Figure 11 contains two more examples for tile figures $E_{I}(5)$ of squid and $E_{I}(5)$ of spider. In the case of squid, the fin part becomes more similar to the goal figure. In the case of spider, the shape of the abdomen becomes slightly more rounded. The 3 tile figures in Figure 11 were not included in the top 10 tile figures obtained by the EST with the $E_{I R}$ distance.

As we have explained, tile figures generated with the $E_{I}$ and $E_{I R}$ distances under the same configuration of $(i, k, j)$ tend to be similar. Nevertheless, for some satisfactory tile figures obtained with the $E_{I R}$ distance, similar ones cannot be obtained using the $E_{I}$ distance. Such tile figures typically contain relatively large deformations, and tile figures $E_{I R}(10)$ of cat, $E_{I R}(7)$ of gecko, and $E_{I R}(6)$ of deer presented in Figure 10 fall into this case. In tile figure $E_{I R}(10)$ of cat, the tail is bent and the face is turned downward, but this appears to be a natural deformation of the goal figure. In tile figure $E_{I R}(7)$ of gecko, the torso part is shortened, but this deformation appears to be essential for representing the detailed structures of the feet. In tile figure $E_{I R}(6)$ of deer, a deer of the goal figure appears to be sitting. Figure 12 shows tile figures optimized using the $E_{I}$ distance under the same configurations of $(i, k, j)$ that generate the three tile figures described above; compared to the tile figures obtained with the $E_{I R}$ distance, these tile figures contain apparently unnatural deformations. Figure 13 shows other interesting tile figures that can only be obtained using the $E_{I R}$ distance.

We conducted an additional questionnaire of 13 people to determine which of the tile figures displayed in Figures 9 and 10 resulted from the most natural deformation. For each goal figure, the tile figures that received the most votes were $E_{I}(7)$ of squid, $E_{I R}(1)$ of cat, $E_{I}(4)$ of dragon, $E_{I R}(9)$ of spider, $E_{I R}(8)$ of gecko, and $E_{I R}(3)$ of deer. Finally, Figure 14 shows the tilings created from the most satisfactory tile figures selected by the authors for all goal figures. See the online supplementary file for other tiling results.

Some readers may be interested to know which IH type the top 10 tile shapes are generated from. Since the order of $K_{i}$ for IH4 is the largest (see the end of Section 2.2), the top 10 tile shapes often belong to IH4. Most of the others would belong to IH5 or IH6 for the same reason, but IH6 tended to be selected more often. For example, 37 of the 51 tile figures displayed in Figures 9, 10, and 13 belong to IH4. Among the remaining tile figures, 13 belong to IH6, which are listed as follows: Euc.(1), $\operatorname{GAD}(8), E_{I}(5)$, and $E_{I}(7)$ of squid; Euc.(3), $G A D(3), E_{I}(1)$, and $E_{I R}(10)$ of cat; $E_{I R}(7)$ of spider; $\mathrm{AD}(3), \mathrm{GAD}(3), E_{I R}(7)$, and $E_{I R}(8)$ of gecko. Only $E_{I R}(1)$ of cat belongs to IH3. IH types of all the top 10 tile shapes are shown in the online supplementary file.

\subsection{Effects of the Heuristic Search}

Table 1 lists the execution time of the EST for the five distance functions. For the GAD, $E_{I}$, and $E_{I R}$ distances, the results of the heuristic search are also listed. For the Euclidean distance, the EST can be performed in a reasonable computation time owing to the speed-up techniques developed in Nagata and Imahori [2020]. For the $\mathrm{AD}$ distance, the same technique was introduced (Section 5), which significantly reduced the required computation time. For example, without this technique, it took $3,525 \mathrm{~s}$ and $12,299 \mathrm{~s}$ for spider and deer, respectively. 


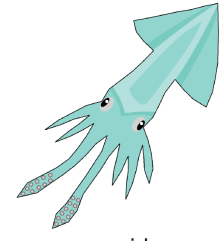

squid

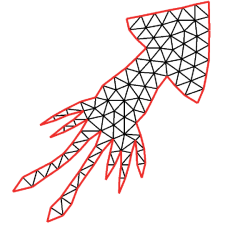

$\left(n=88, n^{\prime}=33\right)$

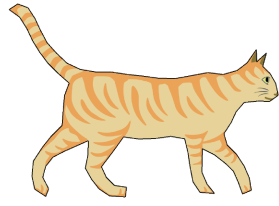

cat

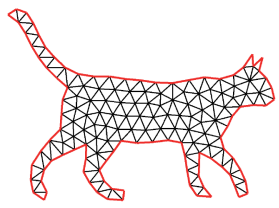

$\left(n=92, n^{\prime}=56\right)$

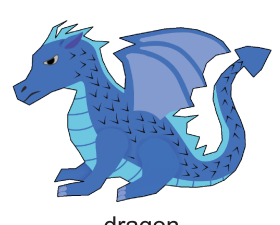

dragon
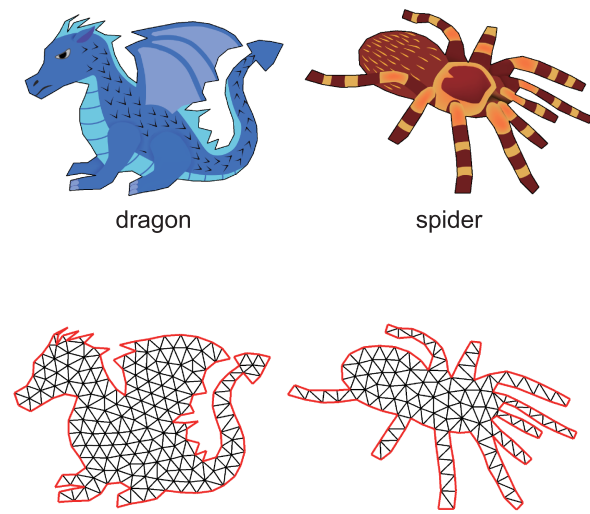

$\left(n=116, n^{\prime}=98\right)$ spider

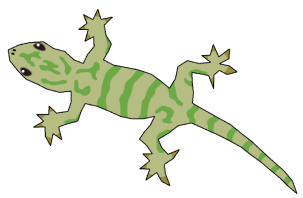

gecko

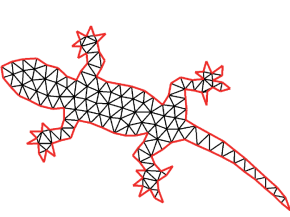

$\left(n=120, n^{\prime}=32\right)$

$\left(n=128, n^{\prime}=38\right)$
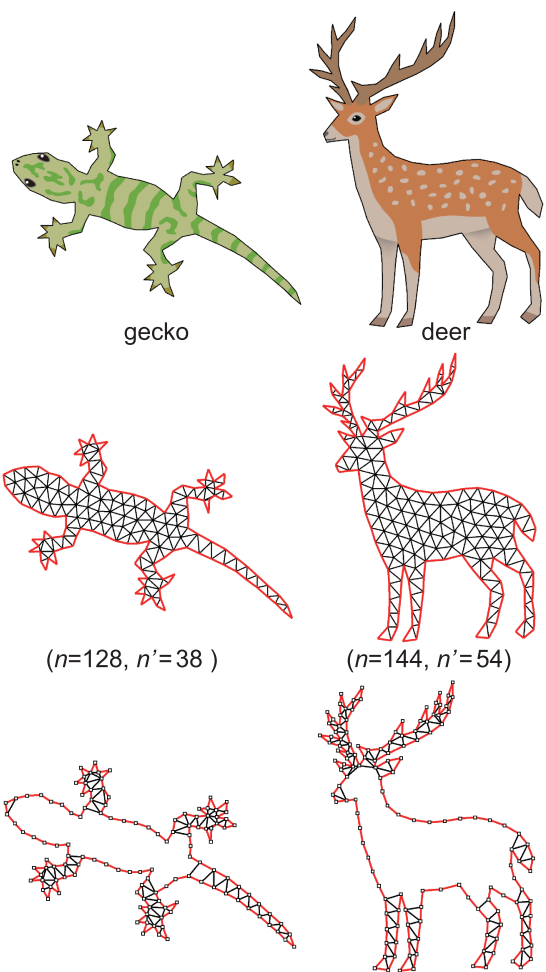
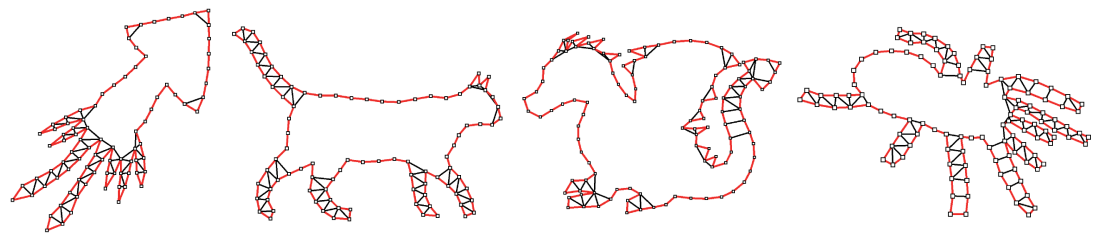

Fig. 8. Six goal figures used in the experiments (top), the corresponding goal meshes (middle), and sets $E_{c}$ of the GAD distance (bottom).

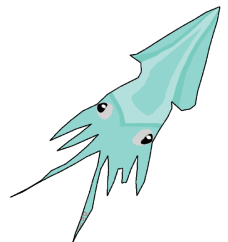

Euc. (1)

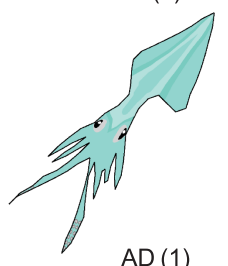

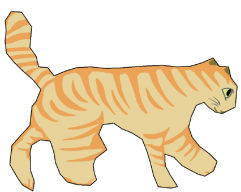

Euc. (3)

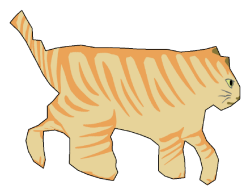

$\mathrm{AD}(3)$

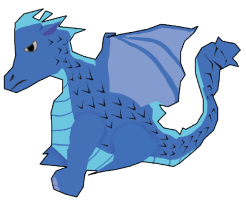

Euc. (1)

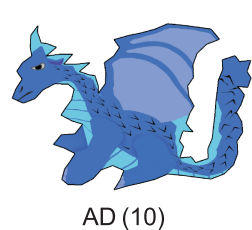

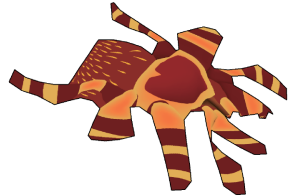

Euc. (8)

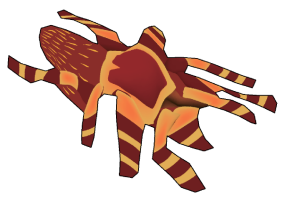

AD (8)

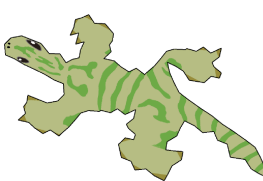

Euc. (4)

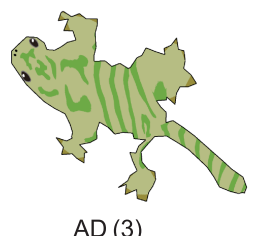

AD (3)
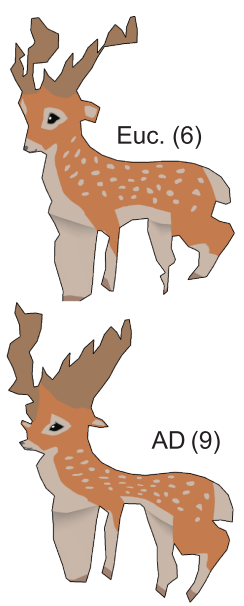

Fig. 9. Tile figures obtained by the EST with the Euclidean and AD distances; the most satisfactory tile figures selected by the questionnaire are presented. The number in parentheses indicates the ranking of the distance value.

For the GAD, $E_{I}$, and $E_{I R}$ distances, the EST is extremely timeconsuming, which makes it problematic in actual use. By contrast, the heuristic search is much faster than the EST, and the results were obtained within $405 \mathrm{~s}$ in the longest case. However, there is a risk of missing some of the top 10 solutions of the EST. For the GAD and $E_{I}$ distances, we confirmed that the heuristic search found all the top 10 solutions except for one $\left(E_{I}(7)\right.$ of deer). For the $E_{I R}$ distance, the heuristic search overlooked some of the top 10 solutions; the numbers of the overlooked solutions were 0 (squid), 2 (cat), 0 (dragon), 1 (spider), 6 (gecko), and 1 (deer). The heuristic search found all tile shapes displayed in Figure 10. However, the three tile shapes shown in Figure 13 were overlooked. ${ }^{6}$ We confirmed that tile shapes with relatively large deformations were likely to be lost in the heuristic search.

${ }^{6}$ If the parameter $n_{A D}$ was increased to 100,000 from $50,000, E_{I R}(7)$ of spider and $E_{I R}(10)$ of deer were found, but the computation time increased by a factor of 1.4 . 

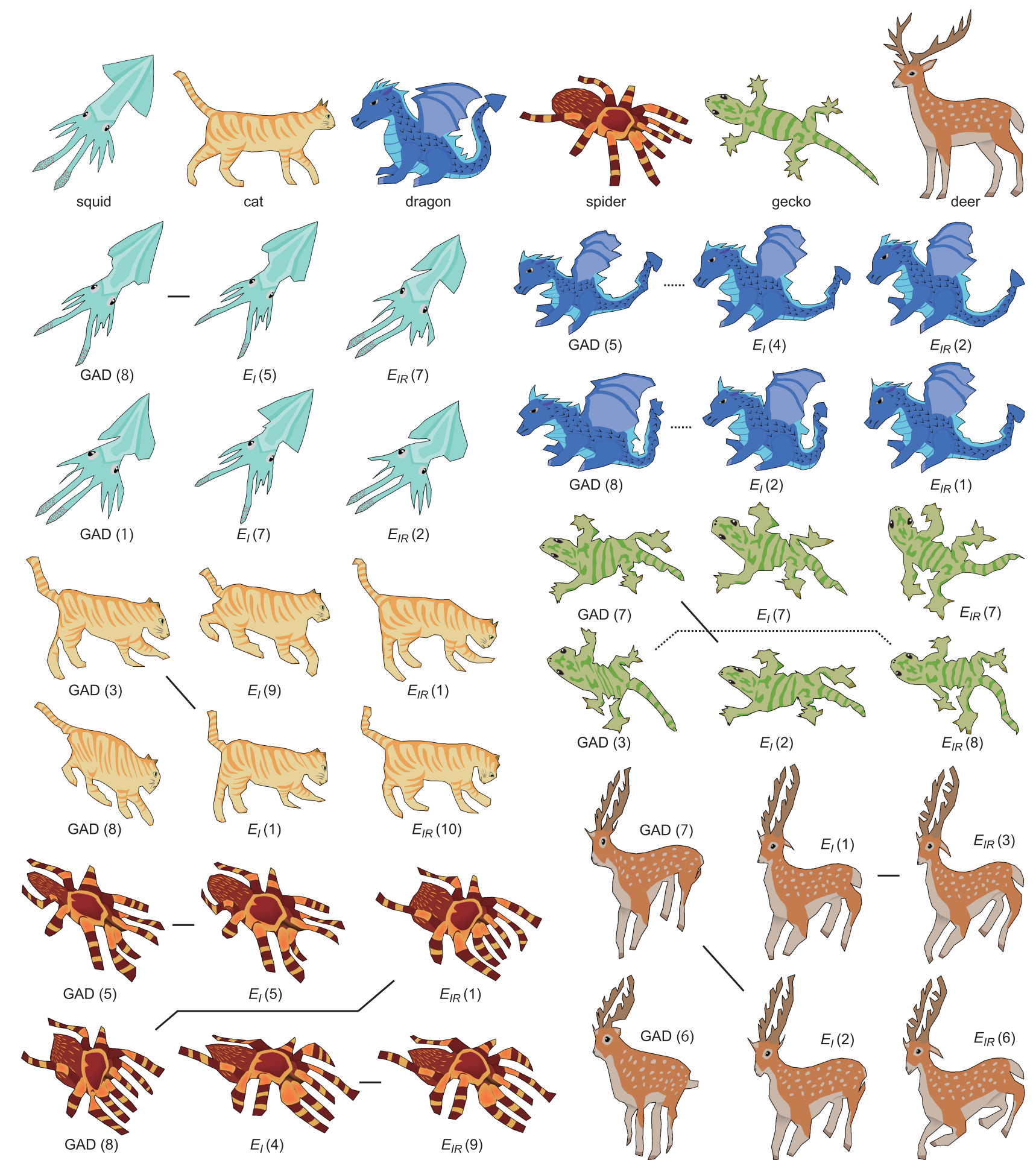

$E_{I R}(1)$
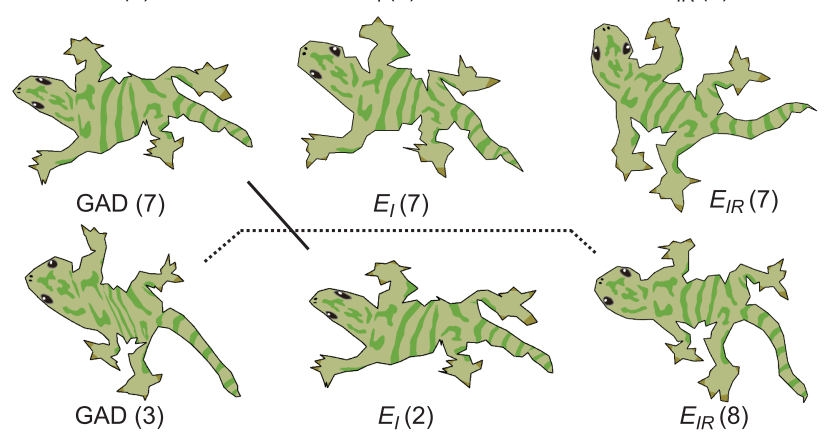

Fig. 10. Tile figures obtained by the EST with the GAD, $E_{I}$, and $E_{I R}$ distances; the most and second most satisfactory tile figures selected by the questionnaire are presented in the upper and lower rows, respectively. The number in parentheses indicates the ranking of the distance value. A solid (dotted) line indicates that the tile figures at both ends are generated under the same (similar) configuration of $(i, k, j)$. The goal figures are displayed again at the top. 

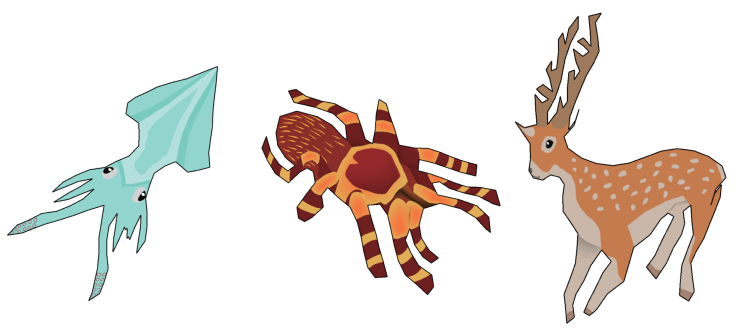

Fig. 11. Tile figures obtained with the $E_{I R}$ distance under the same configurations of $(i, k, j)$ that yield three tile figures $E_{I}(5)$ of squid, $E_{I}(5)$ of spider, and $E_{I}(2)$ of deer presented in Figure 10.
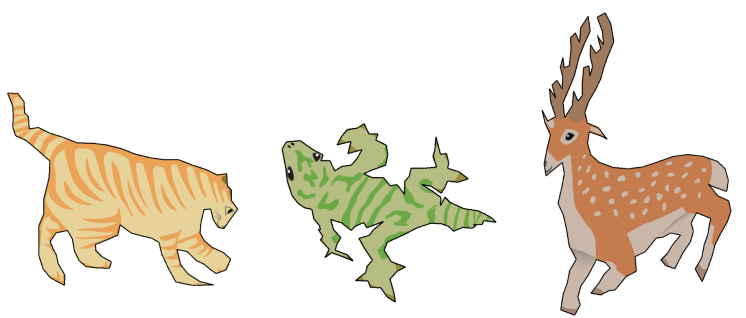

Fig. 12. Tile figures obtained with the $E_{I}$ distance under the same configurations of $(i, k, j)$ that yield three tile figures $E_{I R}(10)$ of cat, $E_{I R}(7)$ of gecko, and $E_{I R}(6)$ of deer presented in Figure 10.
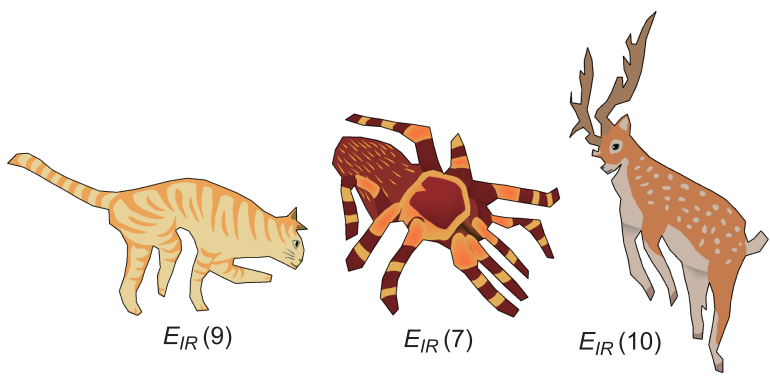

Fig. 13. Other interesting tile figures obtained with the $E_{I R}$ distance.

Table 1. Execution Time in Seconds (or Hours if Specified) of the EST with the Five Distance Functions

\begin{tabular}{c|rrrrrr}
\hline $\begin{array}{c}\text { Distance } \\
n, \boldsymbol{n}^{\prime}\end{array}$ & $\begin{array}{r}\text { Squid } \\
88,33\end{array}$ & $\begin{array}{r}\text { Cat } \\
92,56\end{array}$ & $\begin{array}{r}\text { Dragon } \\
116,98\end{array}$ & $\begin{array}{r}\text { Spider } \\
120,32\end{array}$ & $\begin{array}{r}\text { Gecko } \\
128,38\end{array}$ & $\begin{array}{r}\text { Deer } \\
144,54\end{array}$ \\
\hline \hline Euclidean & 2.8 & 2.7 & 6.9 & 11.1 & 11.4 & 34.4 \\
\hline $\mathrm{AD}$ & 13.0 & 12.7 & 50.2 & 104.1 & 145.0 & 189.3 \\
\hline $\mathrm{GAD}$ & $1.3 \mathbf{h}$ & $1.8 \mathbf{h}$ & $8.3 \mathbf{h}$ & $10.7 \mathbf{h}$ & $15.8 \mathbf{h}$ & $35.4 \mathbf{~ h}$ \\
(heuristic) & 21.3 & 26.6 & 81.7 & 86.2 & 156.2 & 224.6 \\
\hline$E_{I}$ & 1356.9 & $2.6 \mathbf{h}$ & $16.2 \mathbf{h}$ & $5.3 \mathbf{h}$ & $25.9 \mathbf{h}$ & $42.8 \mathbf{~ h}$ \\
(heuristic) & 22.0 & 27.7 & 84.7 & 86.8 & 157.1 & 225.7 \\
\hline$E_{I R}$ & $4.3 \mathbf{h}$ & $7.1 \mathbf{h}$ & $43.1 \mathbf{h}$ & $25.9 \mathbf{h}$ & $43.8 \mathbf{h}$ & $93.7 \mathbf{~ h}$ \\
(heuristic) & 57.9 & 73.3 & 187.5 & 184.3 & 320.4 & 404.9 \\
\hline
\end{tabular}

Results of the heuristic search are also listed.

\section{LIMITATIONS}

During the experiments, there were cases where the use of the $E_{I R}$ distance had a negative effect on the appearance of the obtained tile shapes. A typical example can be seen in the results for the goal figure squid shown in Figure 10, where tile figures $E_{I}(7)$ and $E_{I}(5)$ appear to be better than $E_{I R}(2)$ and $E_{I R}(7)$. This suggests a limitation of the $E_{I R}$ distance, and we analyzed its cause.

If the values of the $E_{I R}$ distance are computed for two tile shapes $E_{I}(7)$ and $E_{I R}(2)$, these values are $76,612.4$, and $40,169.8$, respectively. Why does tile shape $E_{I}(7)$ appear to be more satisfactory than $E_{I R}(2)$ even though the former has a larger value of the $E_{I R}$ distance? Figure 15 shows the mesh representations of the two tile shapes, where the degree of local deformations of cells evaluated by Equation (30) is visualized. In tile mesh $E_{I R}(2)$, the values of Equation (30) are in good agreement with the apparent local deformations. In this sense, the $E_{I R}$ distance is working as expected. In tile mesh $E_{I}(7)$, local deformations that significantly increase the $E_{I R}$ distance value are observed mainly in four locations specified by A, B, C, and D. Although deformation A is noticeable, deformations $\mathrm{B}$ and $\mathrm{C}$ appear to be less noticeable because even if the tips of the leg and tentacle are shortened, the effect on the appearance is minimal. Similarly, deformation D is not noticeable even though two points on the boundary become quite close together because a right-angled curve is maintained in the tile shape. Consequently, tile shape $E_{I}(7)$ is felt to be more satisfactory than $E_{I R}(2)$.

As we have seen in this example, if local deformations are concentrated on those that are not noticeable, more satisfactory tile shapes may be obtained. However, it is difficult to detect such deformations automatically. If we could construct a function to evaluate such deformations, it could be used to re-evaluate the tile shapes obtained by the EST with the $E_{I R}$ distance. Furthermore, if this function can be expressed as a quadratic function of $\tilde{\boldsymbol{u}}$, it can be directly incorporated into the optimization process as a new distance function. The study of this problem is a subject for future research.

As one can easily imagine, the more complex the goal shape is, the more difficult it is to find a satisfactory tile shape. For example, Figure 16 shows the goal shape crab and the most satisfactory tile shapes selected by the authors from the top 10 tile shapes obtained by the EST with the $E_{I}$ and $E_{I R}$ distances. We do not know if there exists a natural deformation of this goal shape that leads to a satisfactory tile shape, but we have not been able to find a satisfactory one, at least with the proposed methods. ${ }^{7}$

\section{DISCUSSION AND CONCLUSIONS}

In this study, a distance function (the $E_{I R}$ distance) developed based on the ARAP deformation scheme was incorporated into the EST for the Escherization problem. The use of the $E_{I R}$ distance yields satisfactory tile shapes (Figure 10), even for complex goal shapes (Figure 8) for which the originally used Euclidean or Procrustes distance does not yield satisfactory results (Figure 9). As expected from the concept of the ARAP deformation scheme, the success is attributed to the ability to generate tile shapes by deforming the goal shape while preserving the local structures over the entire shape. The resulting tile shapes may be significantly deformed from the goal shape, but such deformations can be performed in physically plausible ways. However, the EST with the $E_{I R}$ distance is time-consuming, and we have developed

${ }^{7}$ Escher did draw a successful crab tessellation (sketch \#117 in Escher's notebook), but there is a large gap between his tile shape and our goal shape. 


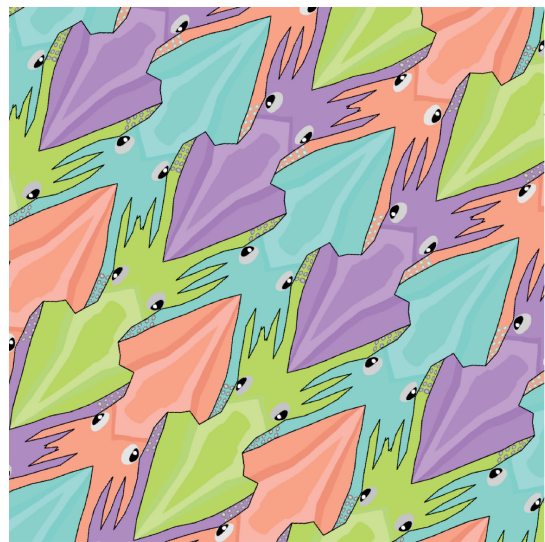

squid: $E_{l}(5)$

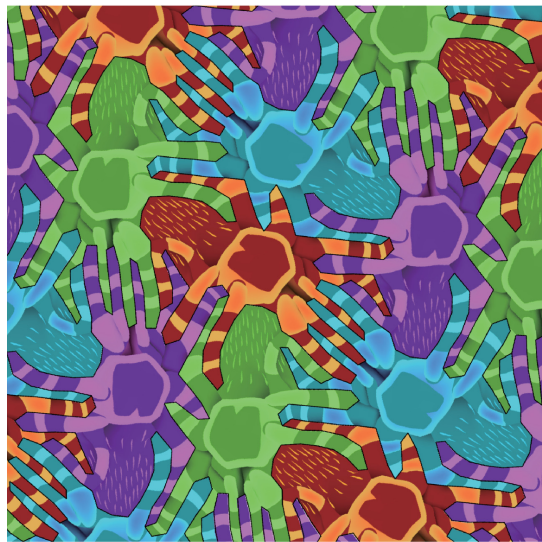

spider: $E_{I R}(7)$

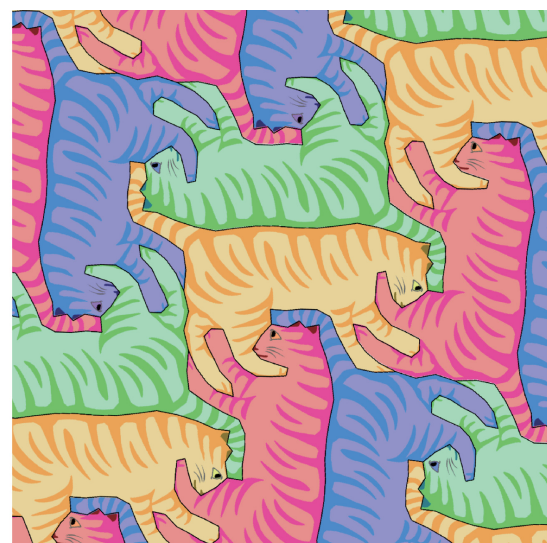

cat: $E_{I R}(10)$

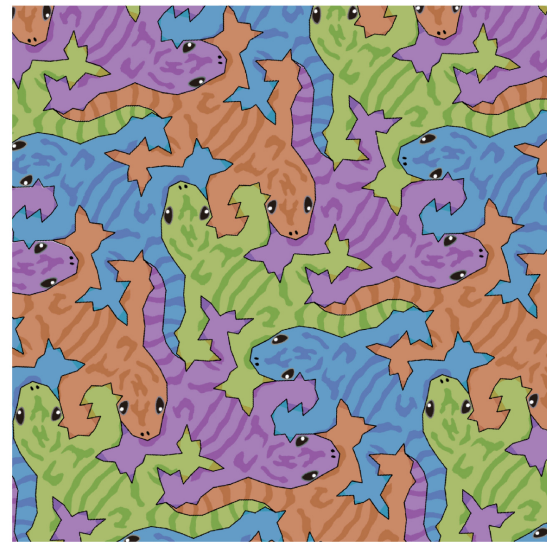

gecko: $E_{I R}(7)$

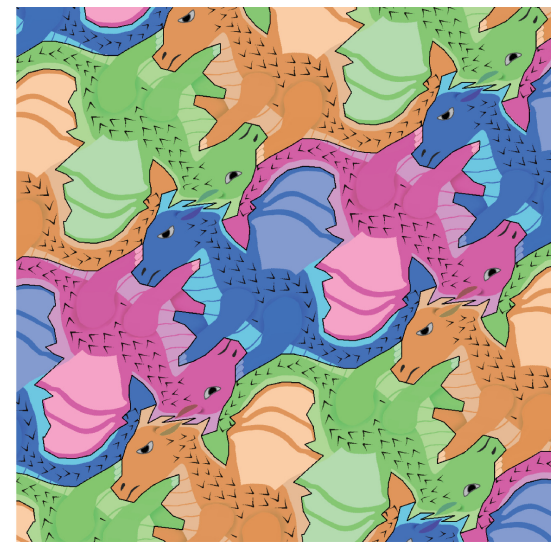

dragon: $E_{l}(2)$

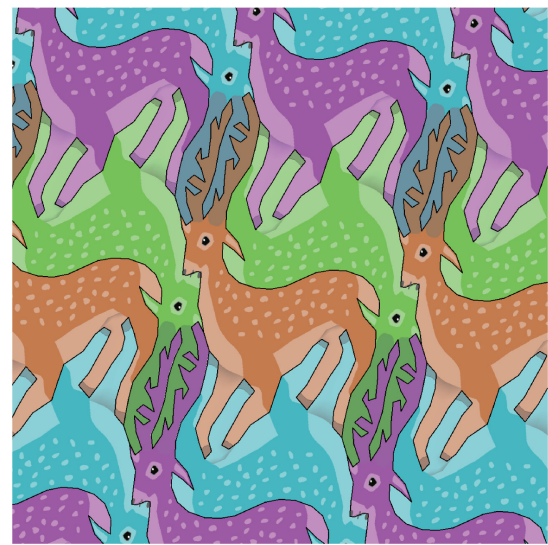

deer: $E_{I R}(3)$

Fig. 14. Tilings created from some of the tile shapes shown in Figure 10.
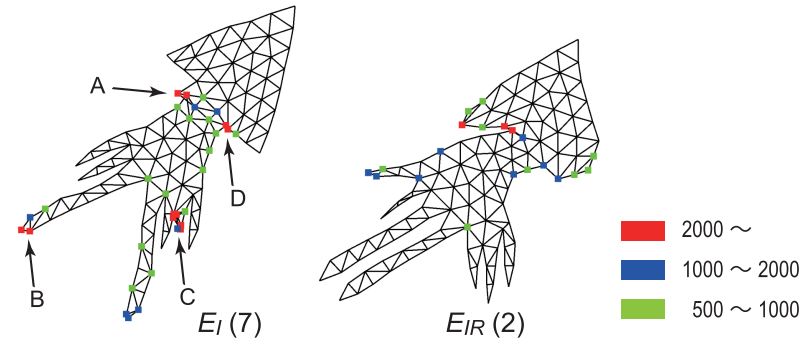

Fig. 15. Visualization of the degree of local deformations evaluated by Equation (30). The centers of cells with large deformations are colored.

a heuristic search algorithm to search for only promising configurations of the templates of the EST. The heuristic search can be completed within a reasonable computation time (Table 1) without losing most of the top-ranked tile shapes obtained by the EST.

The concept of ARAP deformation is well known in 3D surface modeling and deformation, but using this technique in the Escherization problem has effects that are not expected in traditional use. In a typical shape editing task, the primary purpose is to generate a deformed shape that humans expect to see on the target object. On the other hand, the deformation task in the Escherization problem is more challenging because we can hardly imagine what deforma-
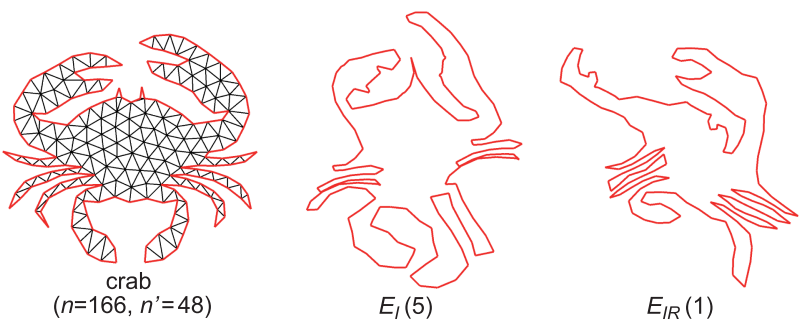

Fig. 16. Tile shapes obtained by the EST with the $E_{I}$ and $E_{I R}$ distances for a further complex goal shape.

tions would be required to generate satisfactory tile shapes. In this sense, the principle of ARAP deformation plays an essential role in creating shapes that humans cannot easily imagine.

From a technical perspective, we applied the ARAP deformation technique to more complex situations than its traditional use. Although both the Escherization problem and typical shape editing tasks are the same in the sense that the original shape is deformed to minimize the energy function under the given constraints, the former is generalized to the case where some of the points of the original shape are constrained by linear equations. We then derived the equations for minimizing the energy function under this 
constraint. In addition, the energy function is further extended to its Procrustes distance version.

\section{APPENDICES}

\section{A DETAILS OF EQUATIONS (16) TO (20)}

First, Equation (17) is derived. We begin by expressing the Procrustes distance $d_{P}^{2}(U, W)$ (Equation (2)) in a different form:

$$
\begin{aligned}
d_{P}^{2}(U, W) & =\min _{\theta}\left(R_{n}(\theta) \boldsymbol{u}-\boldsymbol{w}\right)^{\top}\left(R_{n}(\theta) \boldsymbol{u}-\boldsymbol{w}\right) \\
& =\boldsymbol{u}^{\top} \boldsymbol{u}+\boldsymbol{w}^{\top} \boldsymbol{w}-2 \min _{\theta} \boldsymbol{w}^{\top} R_{n}(\theta) \boldsymbol{u},
\end{aligned}
$$

where $R_{n}(\theta)\left(\in \mathbb{R}^{2 n \times 2 n}\right)$ is the matrix whose non-zero elements are given by filling the $2 \times 2$ block starting at $(2 i-1,2 i-1)$ with $R(\theta)$ for $1 \leq i \leq n$. By comparing Equations (3) and (38), we have

$$
\min _{\theta} \boldsymbol{w}^{\top} R_{n}(\theta) \boldsymbol{u}=\sqrt{\boldsymbol{u}^{\top} V \boldsymbol{u}}
$$

where $V=\boldsymbol{w} \boldsymbol{w}^{\top}+\boldsymbol{w}_{\boldsymbol{c}} \boldsymbol{w}_{\boldsymbol{c}}{ }^{\top}$. The optimal value of $\theta$ is given by $\cos \theta=\frac{\boldsymbol{u}^{\top} \boldsymbol{w}}{\sqrt{\left(\boldsymbol{u}^{\top} \boldsymbol{w}\right)^{2}+\left(\boldsymbol{u}^{\top} \boldsymbol{w}_{c}\right)^{2}}}$ and $\sin \theta=\frac{\boldsymbol{u}^{\top} \boldsymbol{w}_{c}}{\sqrt{\left(\boldsymbol{u}^{\top} \boldsymbol{w}\right)^{2}+\left(\boldsymbol{u}^{\top} \boldsymbol{w}_{c}\right)^{2}}}$.

Using the matrix $R_{n}(\theta), d_{G P}^{2}(U, W)$ (Equation (16)) is expressed and calculated as follows:

$$
\begin{aligned}
d_{G P}^{2}(U, W) & =\min _{\theta}\left(R_{n}(\theta) \boldsymbol{u}-\boldsymbol{w}\right)^{\top} G\left(R_{n}(\theta) \boldsymbol{u}-\boldsymbol{w}\right) \\
& =\boldsymbol{u}^{\top} G \boldsymbol{u}-2 \min _{\theta}(G \boldsymbol{w})^{\top} R_{n}(\theta) \boldsymbol{u}+\boldsymbol{w}^{\top} G \boldsymbol{w}, \\
& =\boldsymbol{u}^{\top} G \boldsymbol{u}-2 \sqrt{\boldsymbol{u}^{\top} G V G \boldsymbol{u}}+\boldsymbol{w}^{\top} G \boldsymbol{w} .
\end{aligned}
$$

To derive Equation (41), $R_{n}(\theta)^{\top} G R_{n}(\theta)=G$ is used; this equality can be understood by considering that the matrix $G$ has the form

$$
\left\{\begin{array}{rl}
{[G]_{2 i-1,2 j-1}} & =[G]_{2 i, 2 j} \\
{[G]_{2 i-1,2 j}} & =[G]_{2 i, 2 j-1}=0
\end{array}(i=1, \ldots, n ; j=1, \ldots, n) .\right.
$$

Using Equation (39), the second term of Equation (41) is calculated by simply replacing $\boldsymbol{w}$ with $G \boldsymbol{w}$ and $\boldsymbol{w}_{c}$ with $G \boldsymbol{w}_{c}$ in this equation. We now have the expression of Equation (17). The optimal value of $\theta$ described in the main text is obtained in the same way.

Next, Equations (19) and (20) are derived. By setting the derivative of the term in Equation (18) equal to zero, we have

$$
B_{i k j}^{\top} G B_{i k j} \xi-\frac{B_{i k j}^{\top} G V G B_{i k j} \xi}{\sqrt{\xi^{\top} B_{i k j}^{\top} G V G B_{i k j} \xi}}=0 .
$$

Therefore, the solution $\xi_{i k j}^{*}$ of the optimization problem (Equation (18)) must be an eigenvector of the generalized eigenvalue problem

$$
B_{i k j}{ }^{\top} G V G B_{i k j} \xi=\lambda B_{i k j}{ }^{\top} G B_{i k j} \xi .
$$

By substituting the left-hand side of Equation (19) into Equation (42), we have

$$
B_{i k j}{ }^{\top} G B_{i k j} \xi=\frac{\lambda B_{i k j}^{\top} G B_{i k j} \xi}{\sqrt{\lambda \xi^{\top} B_{i k j}^{\top} G B_{i k j} \xi}} .
$$

Therefore, the length of an eigenvector $\xi$ is determined by

$$
\xi^{\top} B_{i k j}^{\top} G B_{i k j} \xi=\lambda \text {. }
$$

Let $\xi^{*}$ and $\lambda$ be such an eigenvector and the corresponding eigenvalue. Substituting $\xi^{*}$ into the first and second terms of Equation (18) and using Equations (19) and (44), the sum of these terms is calculated by

$$
\xi^{* \top} B_{i k j}{ }^{\top} G B_{i k j} \xi^{*}-2 \sqrt{\xi^{* \top} B_{i k}^{\top} G V G B_{i k} \xi^{*}}=-\lambda .
$$

Consequently, the minimum value of the optimization problem (Equation (18)) is given by

$$
\operatorname{dist}_{i k j}^{G P}=-\lambda_{i k j}+\boldsymbol{w}^{\top} G \boldsymbol{w}
$$

where $\lambda_{i k j}$ is the maximum eigenvalue of the generalized eigenvalue problem (Equation (19)). The solution $\xi_{i k j}^{*}$ is then given by the corresponding eigenvector.

\section{B PROCRUSTES DISTANCE VERSIONS OF $E_{I}$ AND $E_{I P}$}

First, the Procrustes distance version of $E_{I}(\boldsymbol{u})$ (Equation (27)), denoted as $E_{I P}(\boldsymbol{u})$, is derived. Using the matrix $R_{n}(\theta)$, it is defined as follows:

$$
\begin{aligned}
E_{I P}(\boldsymbol{u}) & =\min _{\theta} E_{I}\left(R_{n}(\theta) \boldsymbol{u}\right) \\
& =\min _{\theta}\left(R_{n}(\theta) \boldsymbol{u}-\boldsymbol{w}\right)^{\top}\left(G_{0}-G_{1} G_{2}^{-1} G_{1}^{\top}\right)\left(R_{n}(\theta) \boldsymbol{u}-\boldsymbol{w}\right) .
\end{aligned}
$$

Therefore, $E_{I P}(\boldsymbol{u})$ has the same form of the distance function $d_{G P}^{2}(U, W)$ (Equation (17)), with $G$ being replaced with $G_{I}=$ $G_{0}-G_{1} G_{2}{ }^{-1} G_{1}^{\top}$.

Next, we consider the Procrustes distance version of $E_{I R}(\boldsymbol{u})$ (Equation (35)), denoted as $E_{I R P}(\boldsymbol{u})$. Using the matrix $R_{n}(\theta)$, it is defined and calculated as follows:

$$
\begin{aligned}
& E_{I R P}(\boldsymbol{u})=\min _{\theta} E_{I R}\left(R_{n}(\theta) \boldsymbol{u}\right) \\
& =\boldsymbol{u}^{\top} G_{I} \boldsymbol{u}-2 \min _{\theta}(H \tilde{\boldsymbol{w}})^{\top} R_{n}(\theta) \boldsymbol{u}+\tilde{\boldsymbol{w}}^{\top}\left(\tilde{G}-T^{\prime \top} G_{2}^{-1} T^{\prime}\right) \tilde{\boldsymbol{w}} \\
& =\boldsymbol{u}^{\top} G_{I} \boldsymbol{u}-2 \sqrt{\boldsymbol{u}^{\top} H \tilde{V} H^{\top} \boldsymbol{u}}+\tilde{\boldsymbol{w}}^{\top}\left(\tilde{G}-T^{\prime \top} G_{2}^{-1} T^{\prime}\right) \tilde{\boldsymbol{w}}
\end{aligned}
$$

where $H=T-G_{1} G_{2}{ }^{-1} T^{\prime}$ and $\tilde{V}$ is defined later. To derive Equation (47), $R_{n}(\theta)^{\top} G_{I} R_{n}(\theta)=G_{I}$ is used; this equality can be understood in the same way as $R_{n}(\theta)^{\top} G R_{n}(\theta)=G$. Using Equation (39), the second term of Equation (47) is calculated by simply replacing $\boldsymbol{w}\left(\boldsymbol{w}_{c}\right)$ with $H \tilde{\boldsymbol{w}}\left(H \tilde{\boldsymbol{w}}_{c}\right)$ in this equation, where the vector $\tilde{\boldsymbol{w}}_{c}$ is defined for the points of $\tilde{W}$ in the same manner as $\boldsymbol{w}_{c}$. The matrix $\tilde{V}$ is then defined by $\tilde{V}=\tilde{\boldsymbol{w}} \tilde{\boldsymbol{w}}^{\top}+\tilde{\boldsymbol{w}}_{\boldsymbol{c}} \tilde{\boldsymbol{w}} \boldsymbol{c}^{\top}$. In the same way, the optimal value of $\theta$ is given by $\cos \theta=\frac{\boldsymbol{u}^{\top} H \tilde{\boldsymbol{w}}}{\sqrt{\left(\boldsymbol{u}^{\top} H \tilde{\boldsymbol{w}}\right)^{2}+\left(\boldsymbol{u}^{\top} H \tilde{\boldsymbol{w}}_{c}\right)^{2}}}$ and $\sin \theta=\frac{\boldsymbol{u}^{\top} H \tilde{\boldsymbol{w}}_{c}}{\sqrt{\left(\boldsymbol{u}^{\top} H \tilde{\boldsymbol{w}}\right)^{2}+\left(\boldsymbol{u}^{\top} H \tilde{\boldsymbol{w}}_{c}\right)^{2}}}$.

For each configuration $(i, k, j)$, we need to minimize the energy function $E_{I R P}(\boldsymbol{u})$ under the constraint $\boldsymbol{u}=B_{i k j} \xi$. This optimization problem can be solved in the same way as in Equations (17) to (20); i.e., the matrices $G$ and $G V G$ are replaced with $G_{I}$ and $H \tilde{V} H^{\top}$, respectively. Consequently, the optimal solution is obtained by solving the generalized eigenvalue problem

$$
B_{i k j}{ }^{\top} H \tilde{V} H^{\top} B_{i k j} \xi=\lambda B_{i k j}{ }^{\top} G_{I} B_{i k j} \xi .
$$

Let $\lambda_{i k j}$ be the maximum eigenvalue of this generalized eigenvalue problem. The solution $\xi_{i k j}^{*}$ is given by the corresponding eigenvector, whose length is determined by $\xi_{i k j}^{*}{ }^{\top} B_{i k j}{ }^{\top} G_{I} B_{i k j} \xi_{i k j}^{*}=\lambda_{i k j}$. 
The optimal value is then given by

$$
\operatorname{dist}_{i k j}^{I R P}=-\lambda_{i k j}+\tilde{\boldsymbol{w}}^{\top}\left(\tilde{G}-T^{\prime \top} G_{2}^{-1} T^{\prime}\right) \tilde{\boldsymbol{w}} .
$$

\section{THE EFFICIENT EST WITH THE AD DISTANCE}

The $\mathrm{AD}$ distance is defined in Equation (10), where $E_{c}$ is a set of the edges of the goal polygon $W$. More specifically, it is represented by Equation (51):

$$
\begin{aligned}
d_{A D}^{2}(U, W) & =\sum_{i=1}^{n}\left\|\left(\boldsymbol{u}_{i+1}-\boldsymbol{u}_{i}\right)-\left(\boldsymbol{w}_{i+1}-\boldsymbol{w}_{i}\right)\right\|^{2} \\
& =\sum_{i=1}^{n}\left\|\overline{\boldsymbol{u}}_{i}-\overline{\boldsymbol{w}}_{i}\right\|^{2}=\|\overline{\boldsymbol{u}}-\overline{\boldsymbol{w}}\|^{2},
\end{aligned}
$$

where $n+1$ is regarded as 1 and the same applies after this. Recall that the coordinates of the $i$ th point of the tile polygon $U$ and the goal polygon $W$ are defined as $\boldsymbol{u}_{i}=\left(x_{i}, y_{i}\right)^{\top}$ and $\boldsymbol{w}_{i}=\left(x_{i}^{w}, y_{i}^{w}\right)^{\top}$, respectively. Then, we define $\overline{\boldsymbol{u}}_{i}=\left(x_{i+1}-x_{i}, y_{i+1}-y_{i}\right)^{\top}$ and $\overline{\boldsymbol{w}}_{i}=\left(x_{i+1}^{w}-x_{i}^{w}, y_{i+1}^{w}-y_{i}^{w}\right)^{\top}$ for $i=1,2, \ldots n$. In addition, two vectors are defined by $\overline{\boldsymbol{u}}=\left(x_{2}-x_{1}, y_{2}-y_{1}, x_{3}-x_{2}, \ldots, y_{1}-y_{n}\right)$ and $\bar{w}=\left(x_{2}^{w}-x_{1}^{w}, y_{2}^{w}-y_{1}^{w}, x_{3}^{w}-x_{2}^{w}, \ldots, y_{1}^{w}-y_{n}^{w}\right)^{\top}$. Using these definitions, the $\mathrm{AD}$ distance is represented by Equation (52).

The right-hand side of Equation (52) is equivalent to the definition of the Euclidean distance (Equation (4)) with $\boldsymbol{u}$ and $\boldsymbol{w}$ being replaced with $\overline{\boldsymbol{u}}$ and $\overline{\boldsymbol{w}}$, respectively. Therefore, if $\overline{\boldsymbol{u}}$ can be parameterized as $\overline{\boldsymbol{u}}=\bar{B}_{i k j} \xi$ with an appropriate matrix $\bar{B}_{i k j}$ whose column vectors are mutually orthonormal, the optimization problem (Equation (13)) for the $\mathrm{AD}$ distance can be converted into the form of the optimization problem (Equation (7)) for the Euclidean distance, and the minimum value is given by $-\xi_{i k j}^{*}{ }^{\top} \xi_{i k j}^{*}+\overline{\boldsymbol{w}}^{\top} \overline{\boldsymbol{w}}$, where $\xi_{i k j}^{*}=\bar{B}_{i k j}{ }^{\top} \bar{w}$.

The matrix $\bar{B}_{i k j}$ can be constructed as follows. Let $B_{i k j}^{\prime}$ be the matrix obtained from $B_{i k j}$ by cyclically shifting up the row elements by two. The possible values for the vector $\overline{\boldsymbol{u}}$ can be parameterized by $\overline{\boldsymbol{u}}=\left(B_{i k j}^{\prime}-B_{i k j}\right) \xi$. Then, the column vectors of the matrix $B_{i k j}^{\prime}-B_{i k j}$ are linearly transformed into a set of mutually orthonormal vectors to construct $\bar{B}_{i k j}$, where the Gram-Schmidt orthogonalization was used in this process. The vector $\overline{\boldsymbol{u}}$ is now parameterized as $\overline{\boldsymbol{u}}=\bar{B}_{i k j} \xi$.

\section{ACKNOWLEDGMENTS}

We thank the editor and all the reviewers for their insightful comments, which greatly improved the quality of our paper.

\section{REFERENCES}

Marc Alexa. 2003. Differential coordinates for local mesh morphing and deformation Visual Computer 19, 2 (2003), 105-114.
Esther M. Arkin, L. Paul Chew, Daniel P. Huttenlocher, Klara Kedem, and Joseph S.B. Mitchell. 1991. An efficiently computable metric for comparing polygonal shapes. IEEE Transactions on Pattern Analysis and Machine Intelligence 13, 3 (1991), 209-216.

Oscar Kin-Chung Au, Chiew-Lan Tai, Ligang Liu, and Hongbo Fu. 2006. Dual Laplacian editing for meshes. IEEE Transactions on Visualization and Computer Graphics 12, 3 (2006), 386-395.

Branko Grünbaum and Geoffrey Colin Shephard. 1987. Tilings and Patterns. W. H. Freeman and Co.

Fabian Hahn, Bernhard Thomaszewski, Stelian Coros, Robert W. Sumner, and Markus Gross. 2013. Efficient simulation of secondary motion in rig-space. In Proceedings of the 12th ACM SIGGRAPH/Eurographics Symposium on Computer Animation. $165-171$.

Takeo Igarashi, Tomer Moscovich, and John F. Hughes. 2005. As-rigid-as-possible shape manipulation. ACM Transactions on Graphics 24, 3 (2005), 1134-1141.

Shinji Imahori and Shohei Sakai. 2013. A Local Search Algorithm for the Escherization Problem. IPSJ SIG Technical Report Vol. 2013-AL-144 No.14 (in Japanese). 8 pages.

Craig S. Kaplan. 2009. Introductory Tiling Theory for Computer Graphics. Morgan and Claypool.

Craig S. Kaplan and David H. Salesin. 2000. Escherization. In Proceedings of the 27th Annual Conference on Computer Graphics and Interactive Techniques. 499-510.

Junhwan Kim and Fabio Pellacini. 2002. Jigsaw image mosaics. ACM Transactions on Graphics 21, 3 (2002), 657-664.

Hiroshi Koizumi and Kokichi Sugihara. 2011. Maximum eigenvalue problem for Escherization. Graphs and Combinatorics 27, 3 (2011), 431-439.

Kin Chung Kwan, Lok Tsun Sinn, Chu Han, Tien-Tsin Wong, and Chi-Wing Fu. 2016. Pyramid of arclength descriptor for generating collage of shapes. ACM Transactions on Graphics 35, 6 (2016), 229:1-229:12.

Yaron Lipman, Olga Sorkine, Daniel Cohen-Or, David Levin, Christian Rossi, and Hans-Peter Seidel. 2004. Differential coordinates for interactive mesh editing. In Proceedings of the Shape Modeling Applications, 2004. 181-190.

Yuichi Nagata and Shinji Imahori. 2020. An efficient exhaustive search algorithm for the escherization problem. Algorithmica 82, 9 (2020), 2520-2534.

Satoshi Ono, Megumi Kisanuki, Hirofumi Machii, and Kazunori Mizuno. 2014. Creation support for Escher-like tiling patterns by interactive genetic algorithms. In SIGGRAPH Asia 2014 Posters. 9:1.

Satoshi Ono, Megumi Kisanuki, Hirofumi Machii, and Kazunori Mizuno. 2015. Figure pattern creation support for Escher-like tiling by interactive genetic algorithms. In Proceedings of the 18th Asia Pacific Symposium on Intelligent and Evolutionary Systems. 421-432.

Chi-Han Peng, Yong-Liang Yang, and Peter Wonka. 2014. Computing layouts with deformable templates. ACM Transactions on Graphics 33, 4 (2014), 99:1-99:11.

Reza Adhitya Saputra, Craig S. Kaplan, and Paul Asente. 2018. RepulsionPak: Deformation-driven element packing with repulsion forces. In Proceedings of the 44th Graphics Interface Conference. 10-17.

Doris Schattschneider. 2004. M.C. Escher: Visions of Symmetry. Harry N. Abrams.

Olga Sorkine and Marc Alexa. 2007. As-rigid-as-possible surface modeling. In Proceedings of the 5th Symposium on Geometry Processing. 109-116.

Olga Sorkine and Mario Botsch. 2009. Interactive shape modeling and deformation. In Eurographics 2009 (Tutorials). 11-37.

Olga Sorkine, Daniel Cohen-Or, Yaron Lipman, Marc Alexa, Christian Rössl, and HP. Seidel. 2004. Laplacian surface editing. In Proceedings of the 2004 Eurographics/ACM SIGGRAPH Symposium on Geometry Processing. 175-184.

Kokichi Sugihara. 2010. Voronoi-diagram approach to Escher-like tiling. In Proceedings of 2010 International Symposium on Voronoi Diagrams in Science and Engineering. 199-204.

Michael Werman and Daphna Weinshall. 1995. Similarity and affine invariant distances between 2D point sets. IEEE Transactions on Pattern Analysis and Machine Intelligence 17, 8 (1995), 810-814.

Changqing Zou, Junjie Cao, Warunika Ranaweera, Ibraheem Alhashim, Ping Tan, Alla Sheffer, and Hao Zhang. 2016. Legible compact calligrams. ACM Transactions on Graphics 35, 4 (2016), 122:1-122:12.

Received December 2020; revised September 2021; accepted September 2021 